FERMILAB-TM-2395-AD

\title{
Emittance Growth Scaling Laws for crossing systematic space-charge driven sixth-order resonances and random octupole driven fourth-order resonances in FFAGs
}

\author{
X. Pang, F. Wang, X. Wang, S.Y. Lee \\ Department of Physics, Indiana University, Bloomington, IN 47405 \\ K.Y. Ng \\ Fermi National Accelerator Laboratory, P.O. Box 500, Batavia, IL 60510
}

\begin{abstract}
Scaling laws of the emittance growth factor (EGF) for a beam crossing the 6 th order systematic space-charge resonances and the random 4 th order resonance driven by octupoles are obtained by numerical multi-particle simulations. These scaling laws can be important in setting the minimum acceleration rate, and the maximum tolerable resonance strength for the design of the non-scaling fixed-field alternating gradient (FFAG) accelerators.

PACS numbers: 52.59.Sa, 29.27.-a, 95.75.-z
\end{abstract}

\section{Introduction}

Fixed-field alternating gradient accelerators (FFAGs) were first proposed in the 1950's [1], but the idea has soon been abandoned by favoring high energy accelerators with the construction of the Alternating Gradient Synchrotron (AGS) at Brookhaven National Laboratory. Recently the FFAG idea has been revived as high beam power is in demand. The beam pulse repetition rate in an accelerator is determined by the ramping rate of the pulsed guide-field and the achievable voltage in rf cavities. The FFAGs have an important advantage over conventional synchrotrons owing to the fact that the guide field is constant so that the repetition rate can be made considerably higher up to a $\mathrm{kHz}$.

The original scaling FFAG design requires large magnet aperture with high nonlinear magnetic fields in order to maintain constant betatron tunes in the ramping cycle. For example, the $150-\mathrm{MeV}$ FFAG constructed recently in Japan has a radial beam excursion from 4.4 to $5.3 \mathrm{~m}$ in an accelerating cycle from $12 \mathrm{MeV}$ to $150 \mathrm{MeV}$, where the betatron tunes are $\nu_{x} \in(3.69,3.80)$ and $\nu_{z} \in(1.14,1.30)$. The difficulties in 
the magnet design can be partially overcome by the non-scaling design [2], where linear fields are employed leaving the betatron tunes to vary as the beam energy increases. The non-scaling design has been considered as a favorable candidate for the acceleration of the muon beams, where the acceleration rate can be very high because of its small rest mass.

Recently, the non-scaling FFAG has been considered as a proton driver. For example, Ruggiero suggested to use three concentric FFAGs as a proton driver to replace the Brookhaven AGS [3] for reaching a final beam power of more than $10 \mathrm{MW}$. For each FFAG, the beam closed orbit has a radial excursion of less than $18 \mathrm{~cm}$ during the acceleration cycle. The betatron tunes vary from $\nu_{x, z}=(40.0,38.1)$ to $(19.1,9.3)$ during acceleration. As shown in Fig. 1, the ramping cycle will cross both the systematic 4 th and 6th order resonances, $4 \nu_{x}=P, 4 \nu_{z}=P, 2 \nu_{x}+2 \nu_{z}=P, 6 \nu_{x}=P, 6 \nu_{z}=P$, $4 \nu_{x}+2 \nu_{z}=P$, and $2 \nu_{x}+4 \nu_{z}=P$, where $P=136$ is the periodicity of the lattice.

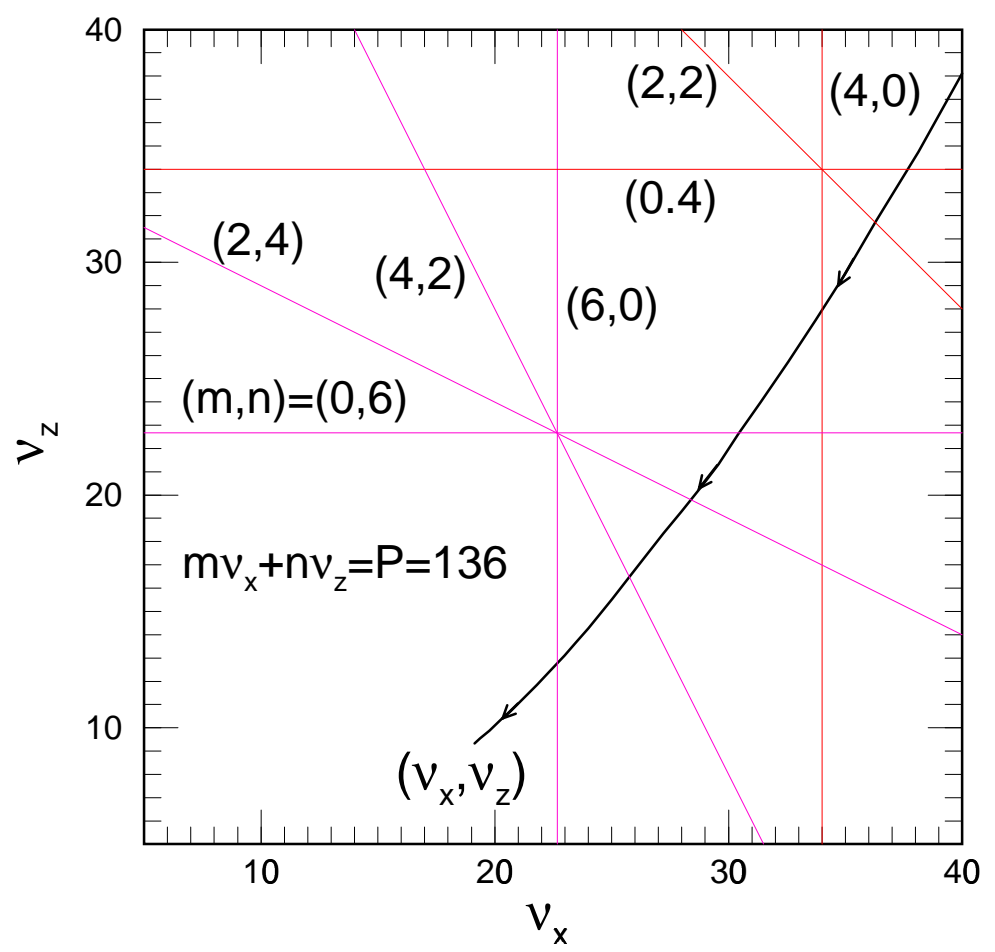

Figure 1. Tune diagram of FFAGs proposed by Ruggiero [3] to replace the Brookhaven AGS. Notice that the betatron tunes of the FFAGs ramp from $\left(\nu_{x}, \nu_{z}\right)=(40.0,38.1)$ to $(19.2,9.3)$ crossing the systematic 4th and 6th order resonances.

For the proton driver in the non-scaling FFAG design, the betatron tune-ramp rate is approximately given by

$$
\frac{\Delta \nu_{x, z}}{\Delta n} \sim-\left(1-\frac{D}{R}\right) \frac{\nu_{x, z}}{2 \beta^{2} E} \frac{\Delta E}{\Delta n},
$$

where $D / R$ is the ratio of dispersion function to the ring's radius and $\Delta E / \Delta n$ is the energy gain per revolution, which depends essentially on the achievable rf voltage in 
cavities. During the acceleration cycle, the beam motion encounters many resonances. The beam quality is an important issue as systematic and random resonances are crossed.

Recently, Lee pointed out that the systematic nonlinear resonances driven by the self-space-charge force may cause substantial emittance growth when the resonances are crossed [4]. He demonstrated a simple scaling property for the emittance growth across the 4th order space-charge resonance. A later article by Lee et al. shows that parametric linear and nonlinear resonances driven by random field errors also lead to emittance growth depending on how fast these resonances are crossed [5].

The purpose of this paper is to continue the investigation of emittance growth on crossing the systematic 6th order resonances as well as the parametric 4th order resonances driven by random nonlinear magnetic fields. We would like to establish scaling laws for these resonances. Given a space-charge tune shift and a resonance strength, we wish to obtain the minimum resonance crossing rate so that emittance growth remains tolerable.

The paper is organized as follows. In Sec. 2, we layout our model of multi-particle simulation calculations. The effect of emittance growth due to crossing the 6th order systematic resonances driven by the self space-charge force is studied in Sec. 3. The effect of random 4 th order resonance driven by octupole-like nonlinearity is examined in Sec. 4. The conclusion is given in Sec. 5 .

\section{The Model}

Our investigation bases mostly on multi-particle simulations. The lattice used here is similar to that of the Fermilab Booster, which can be modeled as 24 superperiod FODO-cells, composing of 48 combined-function F-magnets and 48 combined-function D-magnets. The betatron functions are $\beta_{x, \mathrm{~F}}=40 \mathrm{~m}, \beta_{z, \mathrm{~F}}=8.3 \mathrm{~m}$ at the center of the F-magnets and $\beta_{x, \mathrm{D}}=6.3 \mathrm{~m}, \beta_{z, \mathrm{D}}=21.4 \mathrm{~m}$ at the center of the D-magnets. The dispersion functions are $D_{x, \mathrm{~F}}=4.5 \mathrm{~m}$ and $D_{x, \mathrm{D}}=2.54 \mathrm{~m}$ at the mid-point between two $\mathrm{F}$ and two $\mathrm{D}$ magnets respectively. The betatron tunes will be varied according to the requirement of the simulations. Four-by-four transfer matrices are employed for each period from the center of two D-magnets to the center of the next two F-magnets and from the center of the two F-magnets to the center of the next two D-magnets, thus completing a FODO cell.

The transverse distribution is assumed to be bi-Gaussian all the time. At the end of each revolution turn, the transverse rms beam radii and the position of the beam center are computed from the multi-particle phase space distribution. Then the transverse rms emittances are calculated. These informations are used to compute the spacecharge force, which is applied at each F-magnet set and D-magnet set in the succeeding revolution turn. This procedure has the advantage that the noise in the calculation of the radii is smoothed out in one turn, so that the number of macro-particles used in the simulation, usually 2000, need not be too large. The Gaussian-distribution assumption is certainly not self-consistent. However, this assumption simplifies the space-charge 
force and speeds up the simulations tremendously.

\subsection{Space-Charge Force}

Since the emittance growth rate is usually much faster than a synchrotron period, this justifies the performance of only 2D simulation for a slice of the beam at the longitudinal bunch center. For a beam with linear particle density $N$ and bi-Gaussian charge distribution

$$
\rho(x, z)=\frac{N e}{2 \pi \sigma_{x} \sigma_{z}} e^{-x^{2} / 2 \sigma_{x}^{2}-z^{2} / 2 \sigma_{z}^{2}},
$$

with $\sigma_{x, z}$ being the rms horizontal and vertical beam radii including contribution coming from momentum dispersion, the transverse $2 \mathrm{D}$ space-charge potential is

$$
V_{s c}(x, z)=\frac{K_{s c}}{2} \int_{0}^{\infty} \frac{\exp \left[-\frac{x^{2}}{2 \sigma_{x}^{2}+t}-\frac{z^{2}}{2 \sigma_{z}^{2}+t}\right]-1}{\sqrt{\left(2 \sigma_{x}^{2}+t\right)\left(2 \sigma_{z}^{2}+t\right)}} d t
$$

where

$$
K_{s c}=\frac{2 N r_{0}}{\beta^{2} \gamma^{3}}
$$

is the space-charge perveance, with $r_{0}$ being the particle classical radius and $\beta$ and $\gamma$ the

relativistic parameters. In the simulation, we set, for a bunch containing $N_{\mathrm{B}}$ particles and rms length $\sigma_{s}, N=N_{\mathrm{B}} / \sqrt{2 \pi} \sigma_{s}$. The space-charge force kicking the particles can be obtained by differentiation. Thus each beam particle passing through a length $\ell$ experiences a space-charge kick

$$
\begin{aligned}
& \Delta x^{\prime}=-\frac{\partial V_{s c}}{\partial x} \ell=F_{x, s c} \ell, \\
& \Delta z^{\prime}=-\frac{\partial V_{s c}}{\partial z} \ell=F_{z, s c} \ell .
\end{aligned}
$$

It is straightforward to demonstrate that $F_{x, s c}$ and $F_{z, s c}$ can be expressed analytically in terms of the complex error function. Unfortunately, the application of the analytic expression is cumbersome, because it exhibits an apparent singularity whenever $\sigma_{x}=\sigma_{z}$. The space-charge potential can be expanded as

$$
\begin{aligned}
V_{s c}(x, z)=\quad & -\frac{K_{s c}}{2}\left\{\left[\frac{x^{2}}{\sigma_{x}\left(\sigma_{x}+\sigma_{z}\right)}+\frac{z^{2}}{\sigma_{z}\left(\sigma_{x}+\sigma_{z}\right)}\right]\right. \\
& -\frac{1}{4 \sigma_{x}^{2}\left(\sigma_{x}+\sigma_{z}\right)^{2}}\left[\frac{2+r}{3} x^{4}+\frac{2}{r} x^{2} z^{2}+\frac{1+2 r}{3 r^{3}} z^{4}\right] \\
& +\frac{1}{72 \sigma_{x}^{3}\left(\sigma_{x}+\sigma_{z}\right)^{3}}\left[\frac{8+9 r+3 r^{2}}{5} x^{6}+\frac{3(3+r)}{r} x^{4} z^{2}\right. \\
& \left.\left.+\frac{3(3 r+1)}{r^{3}} x^{2} z^{4}+\frac{8 r^{2}+9 r+3}{5 r^{5}} z^{6}\right]+\cdots\right\},
\end{aligned}
$$

with $r=\sigma_{z} / \sigma_{x}$, where the first term inside the curly brackets represents the linear force, while the second and third terms drive, respectively, the 4th and 6th order resonances. It is not possible, however, to truncate the expansion just up to the order of resonances 
that we wish to study. This is because the truncated space-charge force is accurate only for particles close the beam center and it will increase without limit for particles far away. To solve this problem, an effective space-charge force is introduced for the present investigation:

$$
\begin{aligned}
& F_{x, s c}=\frac{K_{s c} x}{\sigma_{x}\left(\sigma_{x}+\sigma_{z}\right)} \exp \left[-\frac{x^{2}+z^{2}}{\left(\sigma_{x}+\sigma_{z}\right)^{2}}\right], \\
& F_{z, s c}=\frac{K_{s c} z}{\sigma_{z}\left(\sigma_{x}+\sigma_{z}\right)} \exp \left[-\frac{x^{2}+z^{2}}{\left(\sigma_{x}+\sigma_{z}\right)^{2}}\right] .
\end{aligned}
$$

This effective space-charge force reproduces exactly the linear and quadrupole parts of the space-charge force in the round beam geometry, and possesses the property that it vanishes when the particle is far away from the beam center. This effective space-charge force has the disadvantage that it rolls off too fast away from the beam center. Worst of all, it is not derivable from a potential. However, we think that the approximation should be adequate, because $95 \%$ of the particles reside inside two $\sigma$ 's of the beam and emittance growths concerning the crossing of resonances that involve the mixing of the horizontal and vertical phase spaces are not addressed here.

\section{Systematic 6th Order Resonance}

The beam is injected at the rate of $2 \times 10^{11}$ protons per turn. Thus, 100-turn injection implies $2 \times 10^{13}$ particles in the beam or $N_{\mathrm{B}}=2.3 \times 10^{11}$ particles in each of the $84 \mathrm{rf}$ buckets. A bunching factor of $B=0.25$, defined as the ratio of averaged beam current to beam peak current, will be used. Actually, all the discussions in this paper depend on the ratio $N_{\mathrm{B}} / B$ or the maximum linear space-charge tune shifts, but not on $N_{\mathrm{B}}$ and $B$ separately. The injected protons are bi-Gaussian distributed with initial horizontal and vertical normalized rms emittances of $\epsilon_{\mathrm{N}, \mathrm{rms}}=8.33 \times 10^{-6} \pi \mathrm{m}$. The initial bare betatron tunes are $\nu_{x 0}=4.25$ and $\nu_{z 0}=4.30$. Since we are interested only in emittance increase across resonances, the kinetic energy of the beam particles is kept constant at $1 \mathrm{GeV}$ during the tracking, while the betatron tunes are allowed to ramp starting from some specific turn according to some specific ramping rate so that they become $\nu_{x 0}=3.85$ and $\nu_{z 0}=3.90$ at turn 1200 . The emittances at turn 1200 are read and are divided by the initial emittances to give the emittance growth factors (EGFs). In order to minimize all other inference to the space-charge driven systematic resonances, all random fields errors and nonlinear fields in the magnets are turned off.

\subsection{Resonance Strengths}

After Floquet transformation, the terms in the space-charge potential, Eq. (6), responsible for the 6th order resonances can be expressed in terms of action-angle variables in the form

$V_{s c, 6}\left(J_{x}, J_{z}, \psi_{x}, \psi_{z}, \theta\right) \approx-\frac{1}{R} \sum_{\ell}\left|G_{60 \ell}\right| J_{x}^{3} \cos \left(6 \psi_{x}-\ell \theta+\chi_{60 \ell}\right)$ 


$$
\begin{aligned}
& -\frac{1}{R} \sum_{\ell}\left|G_{06 \ell}\right| J_{z}^{3} \cos \left(6 \psi_{z}-\ell \theta+\chi_{06 \ell}\right) \\
& -\frac{1}{R} \sum_{\ell, \pm}\left|G_{4 \pm 2 \ell}\right| J_{x}^{2} J_{z} \cos \left(4 \psi_{x} \pm 2 \psi_{z}-\ell \theta+\chi_{4 \pm 2 \ell}\right) \\
& -\frac{1}{R} \sum_{\ell, \pm}\left|G_{2 \pm 4 \ell}\right| J_{x} J_{z}^{2} \cos \left(2 \psi_{x} \pm 4 \psi_{z}-\ell \theta+\chi_{2 \pm 4 \ell}\right),
\end{aligned}
$$

where $\ell$ is an integer and $R$ is the radius of the accelerator ring. In above, $\left|G_{m n \ell}\right|$ and $\chi_{m n \ell}$ are the amplitude and phase of the resonance strength and they are computed by integrations around the accelerator ring:

$$
\begin{aligned}
G_{60 \ell} & =\frac{1}{5760 \pi} \oint \frac{K_{s c} \beta_{x}^{3}\left(8 \sigma_{x}^{2}+9 \sigma_{x} \sigma_{z}+3 \sigma_{z}^{2}\right)}{\sigma_{x}^{5}\left(\sigma_{x}+\sigma_{z}\right)^{3}} \exp \left[j\left(6 \phi_{x}-6 \nu_{x} \theta+\ell \theta\right)\right] d s, \\
G_{06 \ell} & =\frac{1}{5760 \pi} \oint \frac{K_{s c} \beta_{z}^{3}\left(8 \sigma_{z}^{2}+9 \sigma_{x} \sigma_{z}+3 \sigma_{x}^{2}\right)}{\sigma_{z}^{5}\left(\sigma_{x}+\sigma_{z}\right)^{3}} \exp \left[j\left(6 \phi_{z}-6 \nu_{z} \theta+\ell \theta\right)\right] d s, \\
G_{4 \pm 2 \ell} & =\frac{1}{384 \pi} \oint \frac{K_{s c} \beta_{x}^{2} \beta_{z}\left(3 \sigma_{x}+\sigma_{z}\right)}{\sigma_{x}^{3} \sigma_{z}\left(\sigma_{x}+\sigma_{z}\right)^{3}} \exp \left\{j\left[4 \phi_{x} \pm 2 \phi_{z}-\left(4 \nu_{x} \pm 2 \nu_{z}\right) \theta+\ell \theta\right]\right\} d s, \\
G_{2 \pm 4 \ell} & =\frac{1}{384 \pi} \oint \frac{K_{s c} \beta_{x} \beta_{z}^{2}\left(\sigma_{x}+3 \sigma_{z}\right)}{\sigma_{z}^{3} \sigma_{x}\left(\sigma_{x}+\sigma_{z}\right)^{3}} \exp \left\{j\left[4 \phi_{x} \pm 2 \phi_{z}-\left(4 \nu_{x} \pm 2 \nu_{z}\right) \theta+\ell \theta\right]\right\} d s,
\end{aligned}
$$

where $\phi_{x, z}=\int_{0}^{\theta} d s / \beta_{x, z}$ are the Floquet phase advances. These strengths are to be evaluated just after injection before the crossing of any resonances. There are two contributions to the resonant strengths: space charge and the lattice of the accelerator. For simulation with equal horizontal and vertical emittances to start with, like what we are doing here, the space-charge contribution can be factored out leaving behind the dimensionless reduced resonant strengths $g_{m n \ell}$ according to

$$
G_{m n \ell}=g_{m n \ell} \frac{K_{s c} R}{4 \epsilon_{\mathrm{rms}}^{3}},
$$

where $\epsilon_{\mathrm{rms}}=\epsilon_{\mathrm{N}, \mathrm{rms}} /(\beta \gamma)$ is the unnormalized rms emittance. Here, $K_{s c} R /\left(4 \epsilon_{\mathrm{rms}}\right)$ is just the linear Laslett tune shift for a round beam geometry and $g_{m n \ell}$ depends on the lattice only.

In our simulations, the effective space-charge force of Eq. (7) is used, and these resonant strengths are somewhat different from those in Eq. (9). In our model, spacecharge kicks are applied at each magnet set, or 48 times in a turn. Following the same procedure, these reduced resonant strengths for the systematic resonances $6 \nu_{x}=P$, $6 \nu_{z}=P, 4 \nu_{x}+2 \nu_{z}=P, 2 \nu_{x}+4 \nu_{z}=P$ are found to be $\ddagger$

$$
\left|g_{60 P}\right|=\frac{\epsilon_{\mathrm{rms}}^{3}}{24}\left|\left[\frac{\beta_{x}^{3}}{\sigma_{x}\left(\sigma_{x}+\sigma_{z}\right)^{5}}\right]_{\mathrm{F} \mathrm{mag}}-\left[\frac{\beta_{x}^{3}}{\sigma_{x}\left(\sigma_{x}+\sigma_{z}\right)^{5}}\right]_{\mathrm{D} \mathrm{mag}}\right| \text {, }
$$

$\ddagger$ Since the effective space-force force is not derivable from a potential, $\left|g_{42 P}\right|$ and $\left|g_{24 P}\right|$ can assume alternate forms depending whether they are derived from integration over $x$ or $z$. Thus, numerically they can be $\left|g_{42 P}\right|=1.68 \times 10^{-3}$ or $7.48 \times 10^{-3}$ and $\left|g_{24 P}\right|=2.40 \times 10^{-3}$ or $6.94 \times 10^{-3}$. By the same token, the effective space-charge force gives, for the 4 th order sum resonance, $\left|g_{22 P}\right|=4.03 \times 10^{-2}$ or $3.38 \times 10^{-2}$. Because of this ambiguity, sum resonances are not investigated here with the effective space-charge force. 
Emittance growth scaling laws in resonance crossing

$$
\begin{aligned}
& \left|g_{06 P}\right|=\frac{\epsilon_{\mathrm{rms}}^{3}}{24}\left|\left[\frac{\beta_{z}^{3}}{\sigma_{z}\left(\sigma_{x}+\sigma_{z}\right)^{5}}\right]_{\mathrm{F} \mathrm{mag}}-\left[\frac{\beta_{z}^{3}}{\sigma_{z}\left(\sigma_{x}+\sigma_{z}\right)^{5}}\right]_{\mathrm{D} \mathrm{mag}}\right|, \\
& \left|g_{42 P}\right|=\frac{\epsilon_{\mathrm{rms}}^{3}}{8}\left|\left[\frac{\beta_{x}^{2} \beta_{z}}{\sigma_{x}\left(\sigma_{x}+\sigma_{z}\right)^{5}}\right]_{\mathrm{F} \mathrm{mag}}-\left[\frac{\beta_{x}^{2} \beta_{z}}{\sigma_{x}\left(\sigma_{x}+\sigma_{z}\right)^{5}}\right]_{\mathrm{D} \mathrm{mag}}\right|, \\
& \left|g_{24 P}\right|=\frac{\epsilon_{\mathrm{rms}}^{3}}{8}\left|\left[\frac{\beta_{x} \beta_{z}^{2}}{\sigma_{z}\left(\sigma_{x}+\sigma_{z}\right)^{5}}\right]_{\mathrm{F} \mathrm{mag}}-\left[\frac{\beta_{x} \beta_{z}^{2}}{\sigma_{z}\left(\sigma_{x}+\sigma_{z}\right)^{5}}\right]_{\mathrm{D} \mathrm{mag}}\right| .
\end{aligned}
$$

Since the horizontal and vertical emittances are equal, they can further be simplified to, with neglect of momentum width,

$$
\begin{aligned}
& \left|g_{60 P}\right|=\frac{1}{24}\left|\left[\frac{1}{(1+r)^{5}}\right]_{\mathrm{F} \mathrm{mag}}-\left[\frac{1}{(1+r)^{5}}\right]_{\mathrm{D} \mathrm{mag}}\right|, \\
& \left|g_{06 P}\right|=\frac{1}{24}\left|\left[\frac{1}{\left(1+r^{\prime}\right)^{5}}\right]_{\mathrm{F} \mathrm{mag}}-\left[\frac{1}{\left(1+r^{\prime}\right)^{5}}\right]_{\mathrm{D} \mathrm{mag}}\right|, \\
& \left|g_{42 P}\right|=\frac{1}{8}\left|\left[\frac{r^{2}}{(1+r)^{5}}\right]_{\mathrm{F} \mathrm{mag}}-\left[\frac{r^{2}}{(1+r)^{5}}\right]_{\mathrm{D} \text { mag }}\right|, \\
& \left|g_{24 P}\right|=\frac{1}{8}\left|\left[\frac{r^{\prime 2}}{\left(1+r^{\prime}\right)^{5}}\right]_{\mathrm{F} \mathrm{mag}}-\left[\frac{r^{\prime 2}}{\left(1+r^{\prime}\right)^{5}}\right]_{\mathrm{D} \text { mag }}\right|,
\end{aligned}
$$

where $r=1 / r^{\prime}=\sigma_{z} / \sigma_{x}=\sqrt{\beta_{z} / \beta_{x}}$. They are indeed dependent on the lattice functions only as promised. For our model, numerical evaluation gives $\oint\left|g_{60 P}\right|=6.15 \times 10^{-3}$, $\left|g_{06 P}\right|=4.65 \times 10^{-3},\left|g_{42 P}\right|=1.68 \times 10^{-3},\left|g_{24 P}\right|=2.40 \times 10^{-3}$, which are to be

compared with the 4 th order resonant strengths $\left|g_{40 P}\right|=7.02 \times 10^{-2},\left|g_{04 P}\right|=6.04 \times 10^{-2}$, $\left|g_{22 P}\right|=4.03 \times 10^{-2}$. Due to statistical fluctuation, the horizontal and vertical emittances will be slightly different, and these resonant strengths will be slightly different accordingly. Because of the separation, we can adjust the magnitudes of the reduced resonant strengths as necessary by assigning different values for the betatron functions $\beta_{x, z}$ at the space-charge kick locations. It is important to point out that the model employed here is independent of the radius of the accelerator ring.

The investigation reported in this paper does not include momentum width. When momentum width is included, however, the separation of lattice dependency from space charge is not so exact. But the separation should remain good provided that the momentum width is much smaller than the betatron emittance.

\subsection{Tracking}

Tracking has been performed with 2000 macro-particles for 1200 turns. Figure 2 shows a sample tracking with 100-turn injection at bare tunes $\nu_{x 0}=4.30$ and $\nu_{z 0}=4.25$. Starting from turn 200, the tunes are ramped downwards linearly at the rate of 0.0004

$\S$ Using the exact space-charge potential of Eq. (6), these strengths are $\left|g_{60 P}\right|=1.81 \times 10^{-3}$, $\left|g_{06 P}\right|=1.71 \times 10^{-3},\left|g_{42 P}\right|=1.27 \times 10^{-3}\left|g_{24 P}\right|=1.81 \times 10^{-3},\left|g_{40 P}\right|=2.85 \times 10^{-2},\left|g_{04 P}\right|=2.74 \times 10^{-2}$, $\left|g_{22 P}\right|=0.33 \times 10^{-2}$. The two-kicks per period approximation is assumed. 

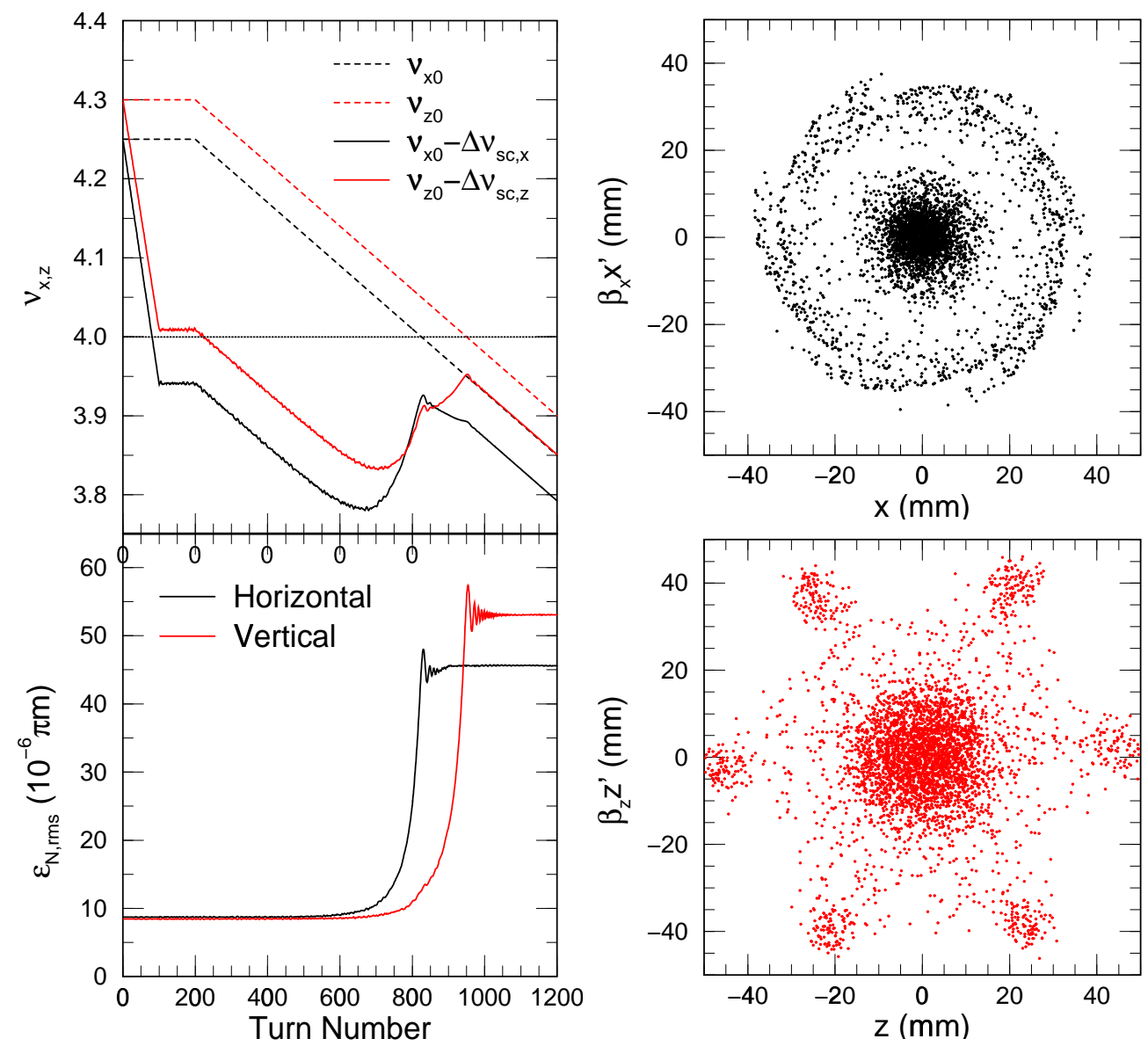

Figure 2. (Color) Top-left: After 100-turn injection at $2.0 \times 10^{11}$ per turn at bunching factor $B=0.25$, bare tunes are ramped downwards from $\left(\nu_{x 0}, \nu_{z 0}\right)=$ $(4.30,4.25)$ starting from turn 200 to $(3.90,3.85)$ at turn 1200 (ramp rate -0.0004 per turn $)$. Systematic resonances $6 \nu_{x 0}=P$ and $6 \nu_{z 0}=P(P=24$ is the lattice periodicity) are crossed at turns 950 and 825, respectively. Bottomleft: Emittance growths are observed when the resonances are crossed. Right: Horizontal and vertical phase-space distributions at turn 900, showing the 6th order resonances.

per turn until they reach 3.90 and 3.85 at turn 1200. The top-left plot shows both the bare tunes and space-charge depressed tunes as functions of turn number, while the bottom-left plot shows the evolution of normalized rms emittances. The systematic resonances $6 \nu_{x 0}=P$ and $6 \nu_{z 0}=P(P=24$, the lattice periodicity $)$ are crossed at turns 950 and 825, respectively, at the moment when the corresponding emittances begin to level off. In the plot $\Delta \nu_{s c, x}$ and $\Delta \nu_{s c, z}$ represent the linear horizontal and vertical spacecharge tune shifts. After injection and before resonances set in, they assume the values $\Delta \nu_{s c, x}=0.309$ and $\Delta \nu_{s c, z}=0.290$. Both the horizontal and vertical emittances start to grow about 150 turns before turns 950 and 825, and become level off after that. As the emittances begin to grow, the beam size increases and the space-charge tune shifts are 
Table 1. Various 6th order resonance strengths arising from assigning different betatron functions at magnets where the space-charge kicks are applied.

\begin{tabular}{cccccc}
\hline$\beta_{x, F}(\mathrm{~m})$ & $\beta_{z, F}(\mathrm{~m})$ & $\beta_{x, D}(\mathrm{~m})$ & $\beta_{z, D}(\mathrm{~m})$ & $\left|g_{60 P}\right|$ & $\left|g_{06 P}\right|$ \\
\hline 40.0 & 8.3 & 6.3 & 21.4 & $6.18 \times 10^{-3}$ & $4.63 \times 10^{-3}$ \\
35.0 & 10.0 & 10.0 & 35.0 & $4.70 \times 10^{-3}$ & $4.67 \times 10^{-3}$ \\
32.0 & 10.0 & 10.0 & 32.0 & $4.29 \times 10^{-3}$ & $4.27 \times 10^{-3}$ \\
25.0 & 10.0 & 10.0 & 25.0 & $3.24 \times 10^{-3}$ & $3.22 \times 10^{-3}$ \\
24.0 & 11.0 & 11.0 & 24.0 & $2.70 \times 10^{-3}$ & $2.69 \times 10^{-3}$ \\
22.0 & 14.0 & 14.0 & 22.0 & $1.51 \times 10^{-3}$ & $1.50 \times 10^{-3}$ \\
22.0 & 15.0 & 15.0 & 22.0 & $1.27 \times 10^{-3}$ & $1.26 \times 10^{-3}$ \\
21.0 & 15.0 & 15.0 & 21.0 & $1.11 \times 10^{-3}$ & $1.11 \times 10^{-3}$ \\
20.0 & 15.0 & 15.0 & 20.0 & $9.48 \times 10^{-4}$ & $9.42 \times 10^{-4}$ \\
18.0 & 15.0 & 15.0 & 18.0 & $5.98 \times 10^{-4}$ & $5.94 \times 10^{-4}$ \\
17.0 & 16.0 & 16.0 & 17.0 & $1.98 \times 10^{-4}$ & $1.97 \times 10^{-4}$ \\
\hline
\end{tabular}

reduced. The reduced resonance strengths are $\left|g_{60 P}\right|=0.00618$ and $\left|g_{06 P}\right|=0.00463$, where $P=24$ is the lattice periodicity. The plots on the right show the particle distribution in the transverse phase spaces at turn 900. Six islands are clearly seen in the bottom-right plot for the vertical phase space just slightly ahead of turn 950 when the 6th order resonance is crossed. The top-right plot shows the horizontal phase space distribution just after crossing the horizontal 6th order resonance. We see that particles are pushed outwards forming an outside ring encircling the inside core, while the 6-fold symmetry is still visible. It is important to point out that it is the bare tunes and not the depressed tunes that determine when systematic resonances are crossed. For all the simulations, no particle loss has been recorded.

\subsection{Scaling}

The tune-ramp rate is increased gradually from $-d \nu_{x 0, z 0} / d n=0.0004$ with start-rampturn-number increasing from 200 in steps of 40 in each simulation until the tune-ramp rate reaches 0.01 with start-ramp-turn-number 1160. For each tune-ramp rate, the resonance strengths are also varied by assigning different values of betatron functions at the space-charge kicks according to Table 1. The emittance growth factor (EGF), which is the ratio of the emittance after to that before crossing the resonance, is computed for each simulation. The results for 100-turn injection with EGF versus tune-ramp rate are depicted in Fig. 3, top plot for the horizontal and bottom plot for the vertical. These are log-log plots. When the EGF is slightly larger than unity, they show linear relations, implying that a scaling power law relationship between EGF and the tune-ramp rate, i.e., $\mathrm{EGF}=(-d \nu / d n)^{-a}$ where $a$ is a constant depending on the resonance strength and the space-charge tune shift. Concentrate on one of these linear relationships, for example the one with $\left|g_{60 P}\right|=0.00324$, the blue data in the top plot of Fig. 3. Extend 
Emittance growth scaling laws in resonance crossing
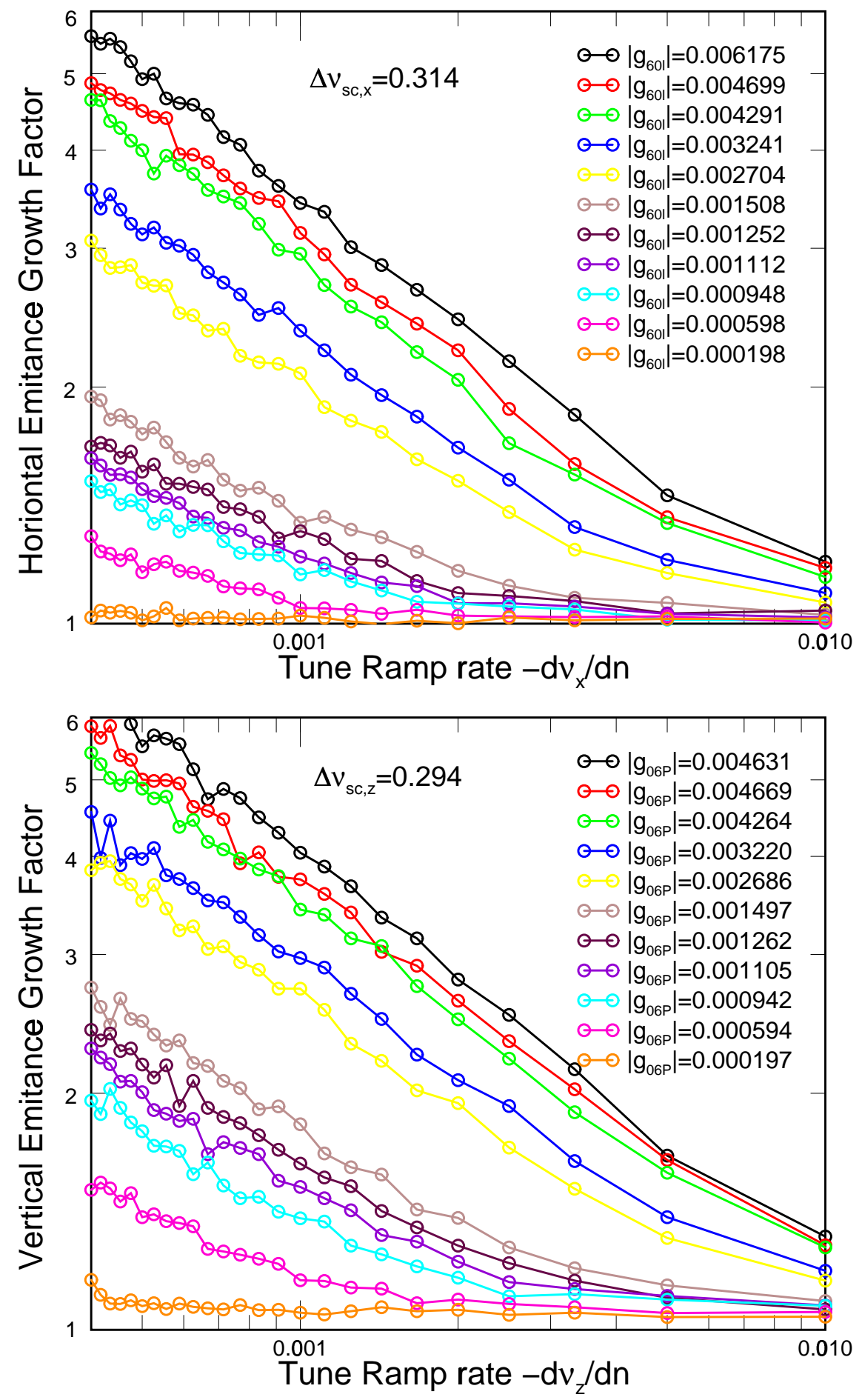

Figure 3. (Color) Horizontal (top) and vertical (bottom) emittance growth factors across the 6 th order systematic resonances $6 \nu_{x 0}=P$ and $6 \nu_{z 0}=P$ ( $P=24$, the lattice periodicity) are plotted as functions of tune-ramp rate for various reduced resonance strengths $g_{60 P}$ and $g_{06 P}$. The bunch intensity is $N_{\mathrm{B}}=23.8 \times 10^{10}$ at bunching factor $B=0.25$ after 100 -turn injection at $2.0 \times 10^{11}$ per turn. 
the linear relationship in the log-log plot to intercept the ramp rate axis at $\mathrm{EGF}=1$ and denote the intercept as the critical tune-ramp rate, which is $\left(-d \nu_{x} / d n\right)_{c} \approx 0.01$. At this critical tune-ramp rate, the curve gives $\mathrm{EGF}=1.18$. We can therefore draw the criterion that if we can tolerate an $\mathrm{EGF} \lesssim 1.2$, the tune-ramp rate must be less than this critical value of 0.01 when the resonance strength is $\left|g_{60 P}\right|=0.003241$. The critical tune-ramp rate is computed in the same way for data belonging to each resonance strength. We find that for space-charge tune shift $\Delta \nu_{s c, x}=0.314$, the power in the power law assumes the value $a=0.56$ at $\left|g_{60 P}\right|=0.00324$ and decreases to $\sim 0.15$ as the resonance strength decreases to 0.00060 . For crossing the resonance $6 \nu_{z 0}=24$, the power law gives $a=0.53$ to 0.23 . The critical tune-ramp rate is now plotted against the resonance strength in Fig. 4, the top for the horizontal and bottom for the vertical.

Similar computations are carried out for different beam intensities. The EGFs for 70-turn injection $\left(N_{\mathrm{B}}=16.7 \times 10^{10}, \Delta \nu_{s c, x}=0.222, \Delta \nu_{s c, z}=0.206\right)$ are plotted in Fig. 5 and that those for 50-turn injection $\left(N_{\mathrm{B}}=11.9 \times 10^{10}, \Delta \nu_{s c, x}=0.160, \Delta \nu_{s c, z}=0.148\right)$ in Fig. 6.

Power law relationships are also evident at these bunch intensities when the EGFs are far from unity. The critical tune-ramp rates are computed and plotted also in Fig. 4. The error bars arise mostly from the uncertainty of straight lines fitting the power laws and the uncertainty of their intercepts on the $d \nu / d n$ axis. The dashed lines in the plots are linear fits to the data. From the plots, the critical tune-ramp rate can be read off once the bunch intensity and 6th order resonance strengths are given. These plots provide a guideline for the design of FFAGs in order to avoid excessive emittance growths while crossing systematic 6 th order resonances. For comparison with the systematic 4 th order resonance, we reproduce in Fig. 7 the relationships between critical tune-ramping rate and resonance strength in Refs. [4] and [5]. We see that the relationships are not linear. However, these results may not be in contradiction with ours. The 6th order resonances are intrinsically weaker than the 4 th, and the maximum $g_{60 P}$ and $g_{06 P}$ studied in this paper have strengths less than one-sixth of the maximum $g_{40 P}$ and $g_{04 P}$ presented in Fig. 7. The maximum critical tune-ramp rate is also about five times smaller. For the smaller ranges of critical tune-ramp rate and $g_{40 P}$, the data in Fig. 7 can also be fitted with linear straight lines.

\subsection{Influence of Sum Resonances}

The space-charge force will couple the two transverse spaces leading to sum resonances. For the simulation depicted in Fig. 2, the two 6th order sum resonances $4 \nu_{x 0}+2 \nu_{z 0}=P$ and $2 \nu_{x 0}+4 \nu_{z 0}=P$ are crossed at turns 866.7 and 908.3. We see, however, only slight increase in the horizontal emittance near turn 908.3 in the emittance plot, but nothing at all near turn 866.7. This may indicate that the resonance strengths for these two resonances are small from our effective space-charge force.\| As was referenced earlier, there is no accurate way to compute the strengths for these two sum resonances

\| This does not necessary imply that the correct driving forces for these two sum resonances are small. 
Emittance growth scaling laws in resonance crossing
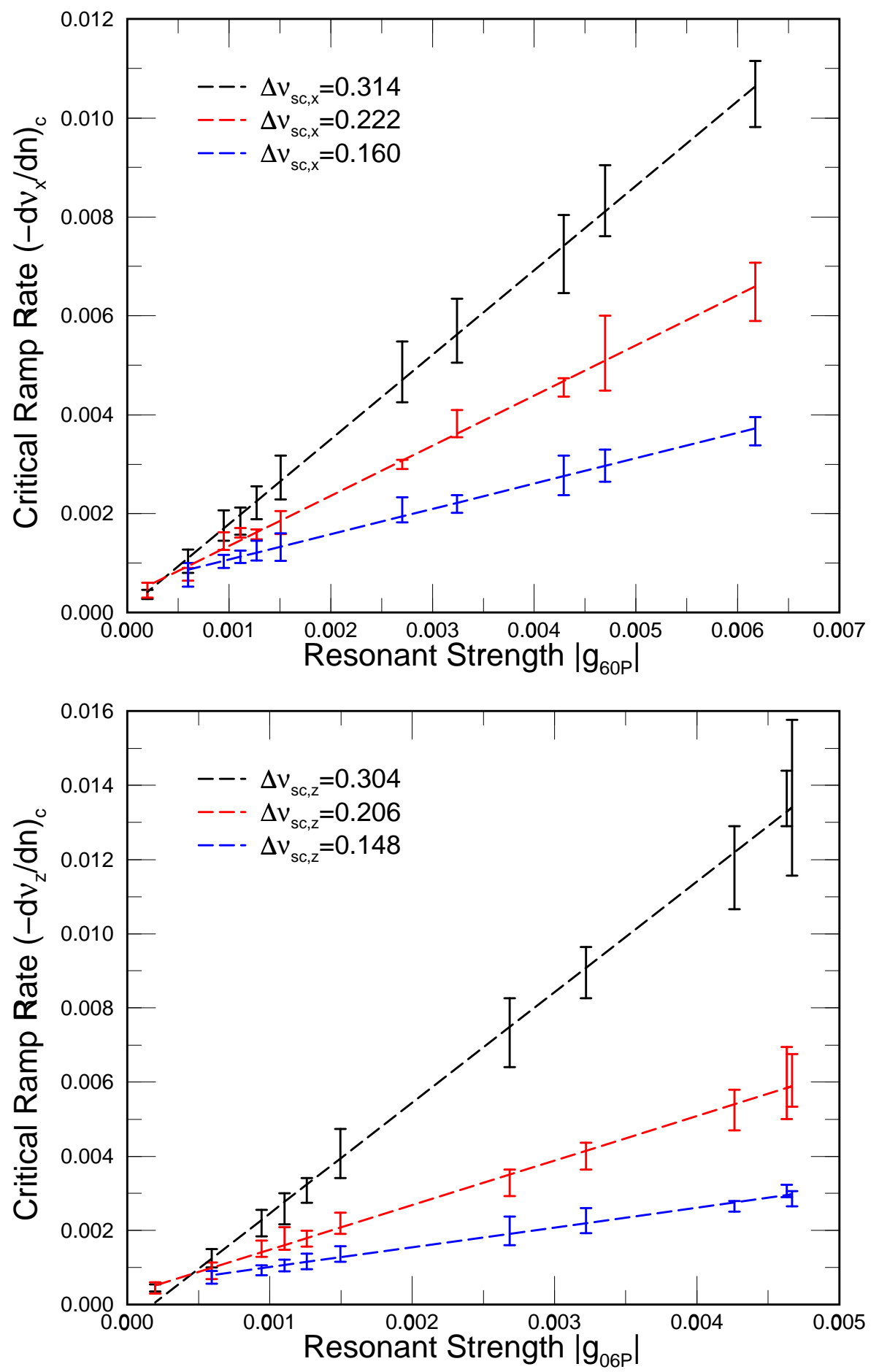

Figure 4. (Color) Top: Critical tune-ramp rates across the systematic 6th order resonance $6 \nu_{x 0}=P(P=24)$ are plotted as functions of reduced resonance strength $\left|g_{60 P}\right|$ for various linear space-charge tune shifts or bunch intensities. Bottom: Same for the resonance $6 \nu_{z 0}=P(P=24$, the lattice periodicity). Dashed lines are linear fits to the data. 
Emittance growth scaling laws in resonance crossing
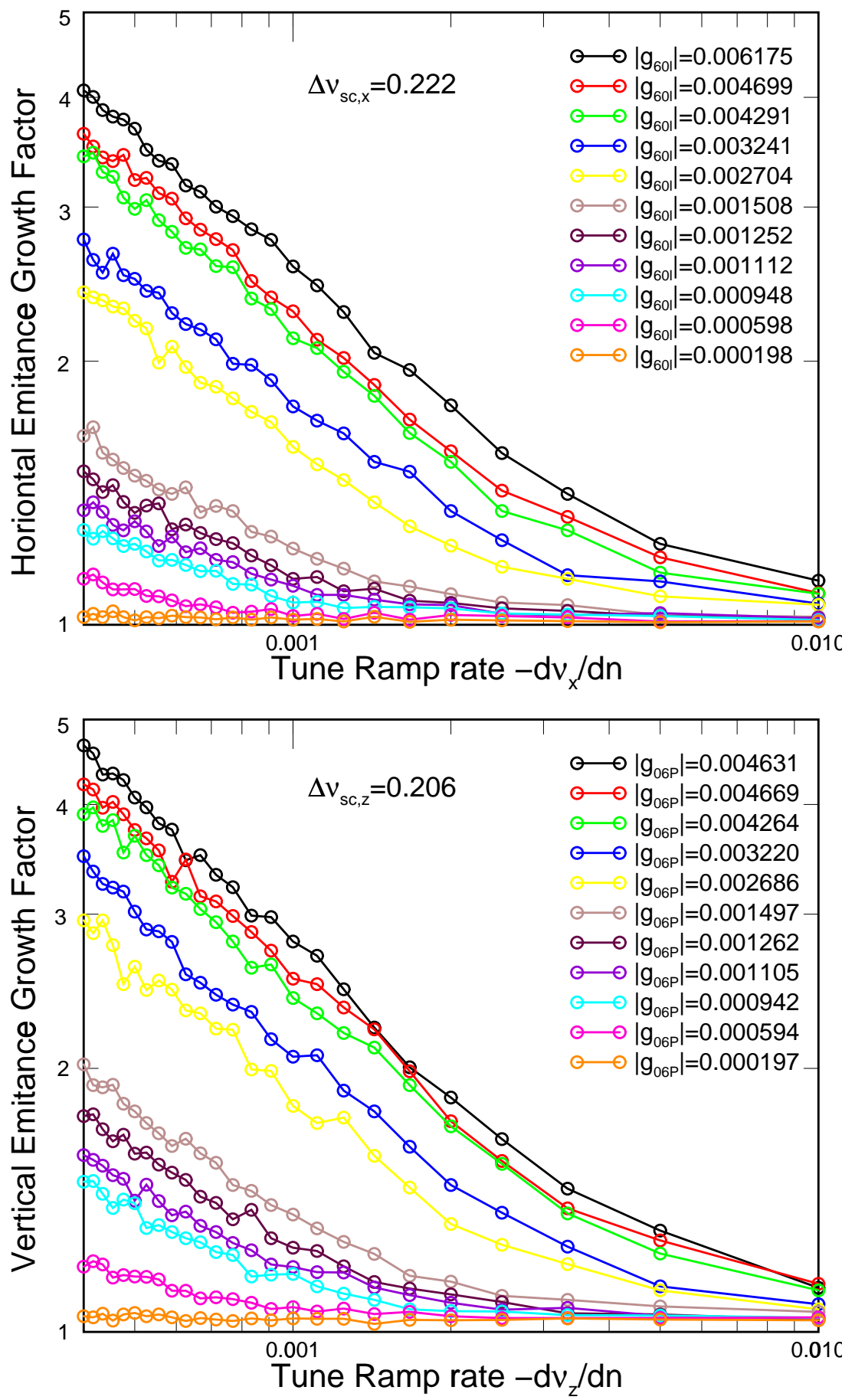

Figure 5. (Color) Horizontal (top) and vertical (bottom) emittance growth factors across the 6 th order systematic resonances $6 \nu_{x 0}=P$ and $6 \nu_{z 0}=P$ ( $P=24$, the lattice periodicity) are plotted as functions of tune ramp rate for various reduced resonance strengths $g_{60 P}$ and $g_{06 P}$. The bunch intensity is $N_{\mathrm{B}}=16.7 \times 10^{10}$ at bunching factor $B=0.25$ after 70 -turn injection of $2.0 \times 10^{11}$ per turn with linear space-charge tune shifts $\Delta \nu_{s c, x}=0.222$ and $\Delta \nu_{s c, z}=0.206$. 

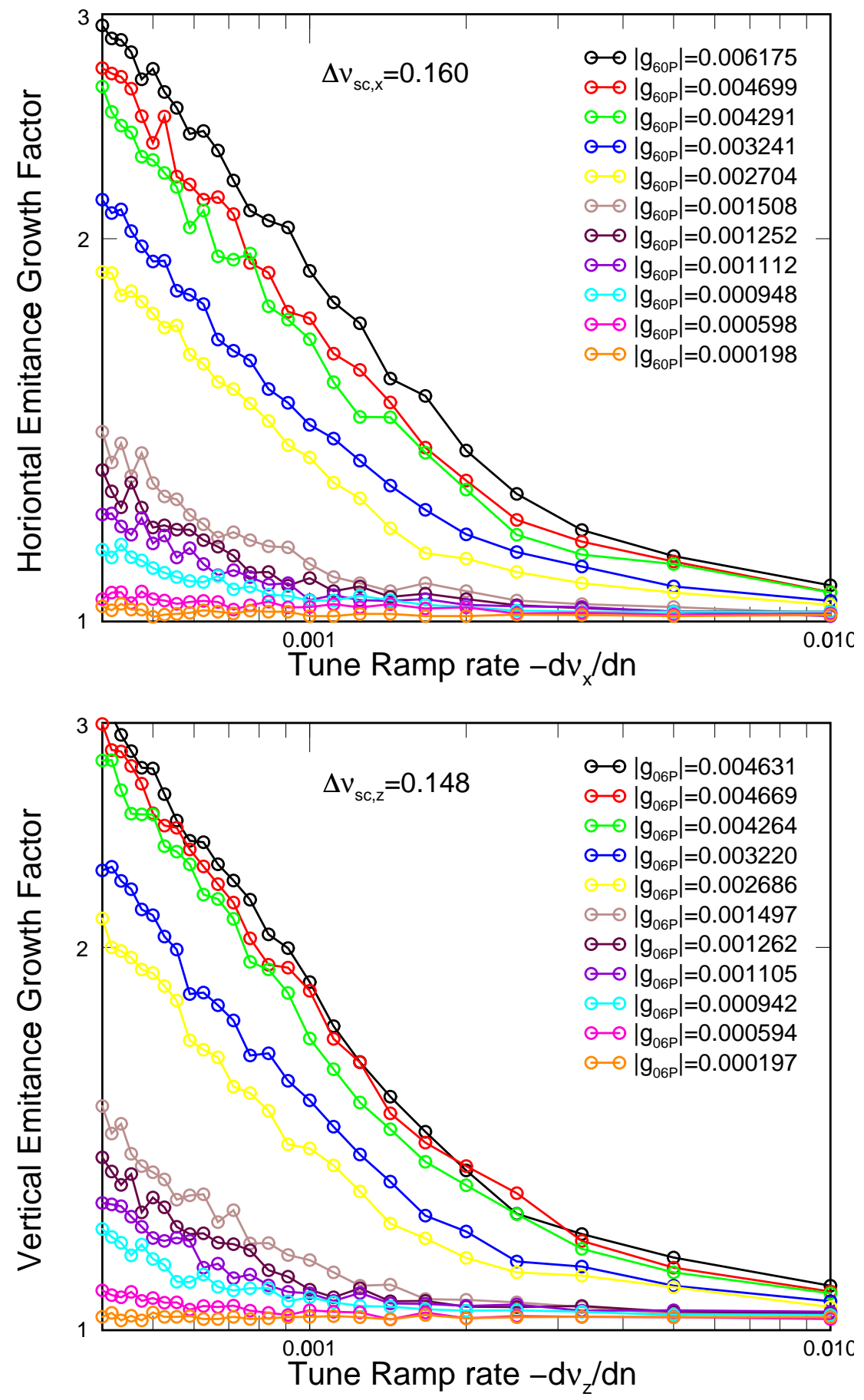

Figure 6. (Color) Horizontal (top) and vertical (bottom) emittance growth factors across the 6 th order systematic resonances $6 \nu_{x 0}=P$ and $6 \nu_{z 0}=P$ $(P=24$, the lattice periodicity) are plotted as functions of tune ramp rate for various reduced resonance strengths $g_{60 P}$ and $g_{06 P}$. The bunch intensity is $N_{\mathrm{B}}=11.9 \times 10^{10}$ at bunching factor $B=0.25$ after 50 -turn injection of $2.0 \times 10^{11}$ per turn with linear space-charge tune shifts $\Delta \nu_{s c, x}=0.160$ and $\Delta \nu_{s c, z}=0.148$. 


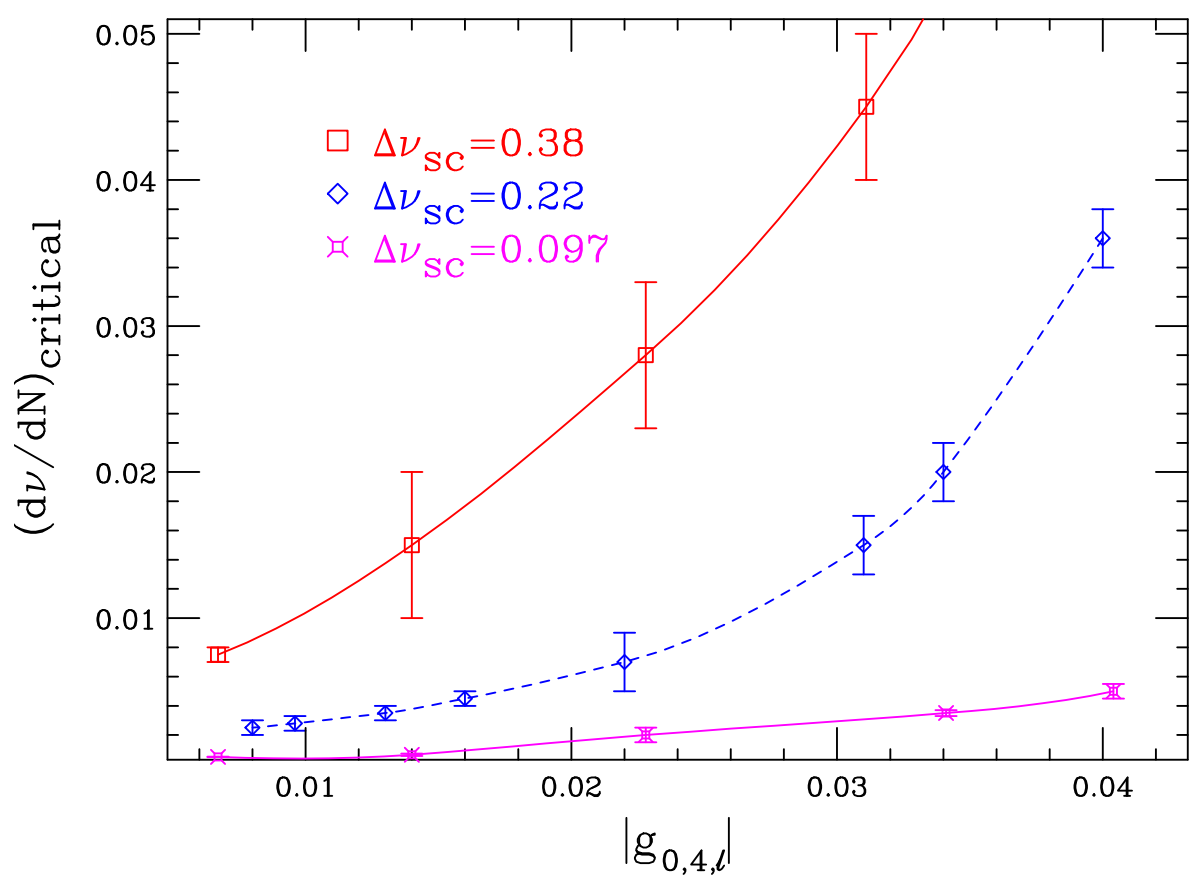

Figure 7. (color) Curves of critical tune-ramp rate versus strength $g_{04 P}$ of 4 th order resonance $4 \nu_{z 0}=24$, reprinted from Refs. [4] and [5]. Note the nonlinear behaviors of the curves. (Courtesy Lee, et al.)

because the effective space-charge force we use is not derivable from a potential. Since the effective space-charge force does not follow from the Hamilton equations, Liouville theorem breaks down when the two transverse phase spaces are coupled. Luckily the effect of the two sum resonances is small in the simulation, and therefore should not interfere much with the emittance growths across $6 \nu_{x 0}=P$ and $6 \nu_{x 0}=P$.

To investigate further about the influence of the sum resonances, we perform a similar simulation with the sum resonances occurring farther away from the two singlespace resonances. Everything is the same as the simulation in Fig. 2 except that the bare vertical tune is raised to $\nu_{z 0}=4.45$ at injection. Both tunes are ramped downward in the same way at turn 200 at the rate of 0.0004 per turn. The beam intensity remains at 100 -turn injection at $2.0 \times 10^{11}$ per turn. The tune and emittance evolutions are shown in the left plots of Fig. 8. The resonance $6 \nu_{x 0}=P$ is first crossed at turn 825 , then the sum resonance $4 \nu_{x 0}+2 \nu_{z 0}=P$ at turn 991.7, then the sum resonance $2 \nu_{x 0}+4 \nu_{z 0}=P$ at turn 1158.3, and finally the resonance $6 \nu_{z 0}=24$ at turn 1325. Just as in Fig. 2, the crossing of the sum resonance $4 \nu_{x 0}+2 \nu_{z 0}=P$ (No. 3 in the emittance plot) just causes very small increases in the horizontal emittance, while the crossing of the other sum resonance $2 \nu_{x 0}+4 \nu_{z 0}=P$ (No. 4) causes slightly larger increases in the emittances. However, the final vertical emittance is smaller than final horizontal emittance. There can be two reasons why the EGF for crossing the resonance $6 \nu_{z 0}=P$ is smaller than that for crossing the resonance $6 \nu_{x 0}=P$. First the reduced resonance strength for the former is smaller than the latter $\left(\left|g_{06 P}\right|=0.00465\right.$ versus $\left.\left|g_{60 P}\right|=0.00615\right)$. Second, resonance 

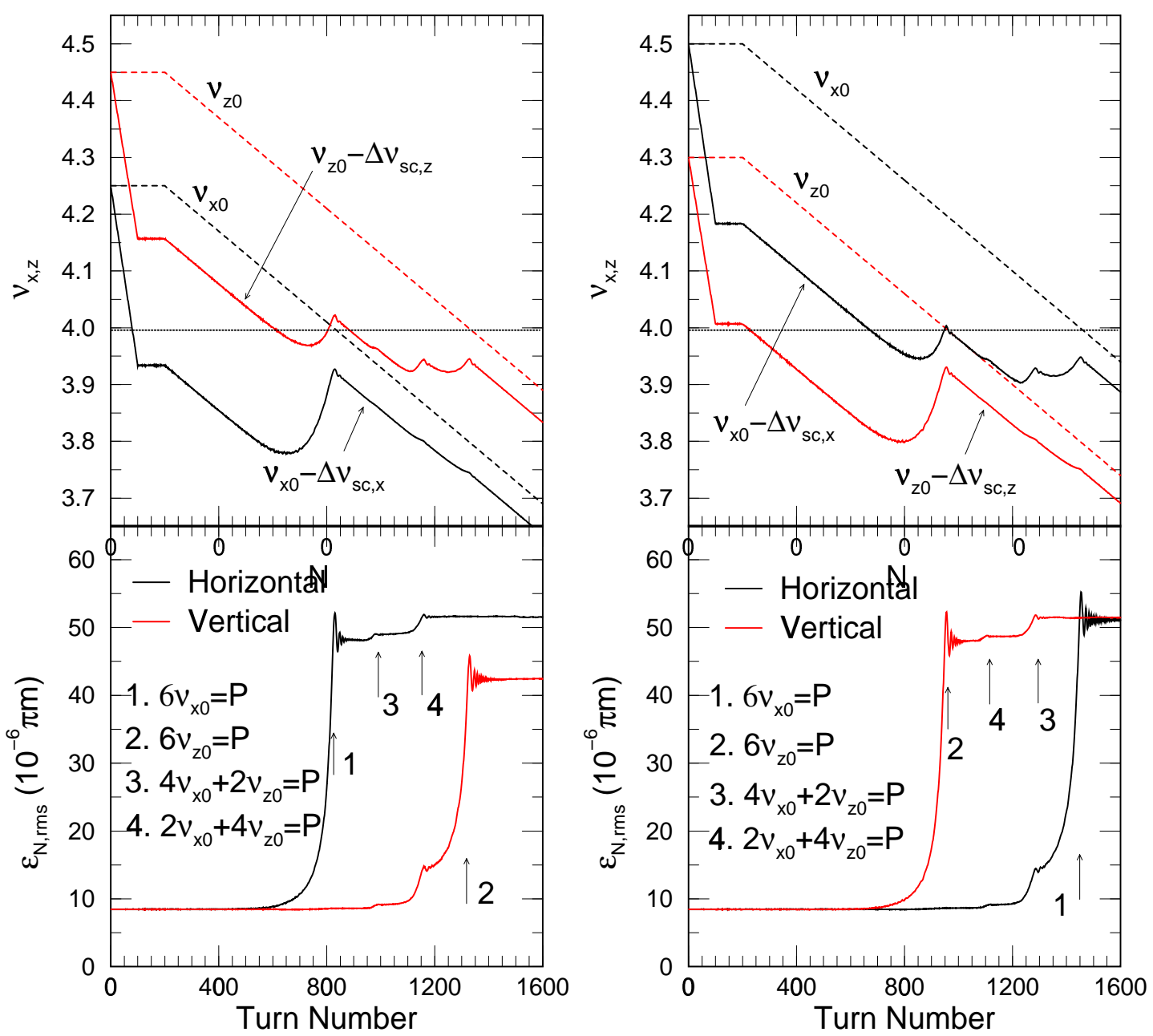

Figure 8. (Color) Top-left: After 100-turn injection at $2.0 \times 10^{11}$ per turn at bunching factor $B=0.25$, bare tunes are ramped downwards from $\left(\nu_{x 0}, \nu_{z 0}\right)=(4.25,4.45)$ from turn 200 at the rate of 0.0004 per turn. Bottomleft: Evolution of normalized rms emittances. Systematic 6th order resonances are crossed at turn 825 for $6 \nu_{x 0}=P$, turn 1325 for $6 \nu_{z 0}=P$, turn 991.7 for $4 \nu_{x 0}+2 \nu_{z 0}=P$, and turn 1158.3 for $2 \nu_{x 0}+4 \nu_{z 0}=P(P=24$ is the lattice periodicity). Right plots are the same simulation, but starting with bare tunes $\left(\nu_{x 0}, \nu_{z 0}\right)=(4.50,4.30)$. The resonances are crossed at turn 1450 for $6 \nu_{x 0}=P$, turn 950 for $6 \nu_{z 0}=P$, turn 1283.3 for $4 \nu_{x 0}+2 \nu_{z 0}=P$, and turn 1116.7 for $2 \nu_{x 0}+4 \nu_{z 0}=P$. 
$6 \nu_{x 0}=P$ is crossed first. With the growth of the horizontal emittance, the horizontal beam size increases and the vertical space-charge force driving the coming resonance $6 \nu_{z 0}=P$ becomes smaller. However, this argument does not work for the simulation in Fig. 2, where the EGF is larger than across the resonance resonance $6 \nu_{z 0}=P$, although this is also the last resonance to cross (final emittance $\epsilon_{\mathrm{N}, \mathrm{rms}}=42.5 \times 10^{-6} \pi \mathrm{m}$ here versus $50.3 \times 10^{-6} \pi \mathrm{m}$ in Fig. 2).

The results of another simulation are shown in the right plots of Fig. 8. The beam intensity and tune-ramp rate are the same as before. The only difference is the choice of initial bare tunes $\nu_{x 0}=4.50$ and $\nu_{z 0}=4.30$. Here in the emittance plot we see resonance $6 \nu_{z 0}=P$ is first crossed at turn 950, followed by resonance $2 \nu_{x 0}+4 \nu_{z 0}=P$ at turn 1116.7, resonance $4 \nu_{x 0}+2 \nu_{z 0}=P$ at turn 1283.3 , and finally $6 \nu_{x 0}=P$ at turn 1450. As in the previous simulation (left plots) the crossing of the second sum resonance initiates larger emittance growth than the first sum resonance. However, the order of crossing the two sum resonances is now reversed. As for the resonance $6 \nu_{z 0}=P$, the emittance growth is larger than in the previous simulation (left plots) because it is the first resonance crossed. We also find the final horizontal emittance to be the same in the two emittance plots and do not understand why it is not smaller because the resonance $6 \nu_{x 0}=P$ is the last crossed and the space-charge force driving the resonance should have been reduced after encountering vertical emittance growth for three resonances.

Next we try another scenario by ramping downwards only one bare tune but holding the other constant. Although this scenario is not realistic in an FFAG, however, we may see from it how the each of the two single-phase-space resonances behaves when the other is not present. The left plots of Fig. 9 are for the simulation with horizontal bare tune held fixed at $\nu_{x 0}=4.25$ while the vertical bare tune is ramped downwards at 0.0004 per turn. The emittance growths across the first resonance $6 \nu_{z 0}=P$ and the first sum resonance $2 \nu_{x 0}+4 \nu_{z 0}=P$ are very similar to what we have demonstrated in previous simulations. The real difference comes from crossing the second sum resonance $4 \nu_{x 0}+2 \nu_{z 0}=P$. Here the emittance growth has been very much larger. For the simulation shown in the right plots of Fig. 9, $\nu_{x 0}$ is ramped downwards and $\nu_{z 0}$ is kept constant. We also see a rather large increase in emittance when the second sum resonance is crossed. It appears that when the crossings of the two single-phase-space resonances $6 \nu_{x 0}=P$ and $6 \nu_{z 0}=P$ are separated farther apart in turn number, the growth in emittance across the second sum resonance will be larger. Whether this phenomenon is real or just fictitious as a result of the non-conservative effective spacecharge force is unclear.

In any case, we can draw a conclusion from these simulations that independent of the separation of turns for crossing $6 \nu_{x 0}=P$ and $6 \nu_{z 0}=P$, the emittance growth factors can differ by at most $\pm 10 \%$. This can be used as the magnitude of the errors in the EGFs when we seek the critical tune-ramp rate by introducing the linear relationship and intercept in the log-log plots of Figs. 3, 5, and 6 . 

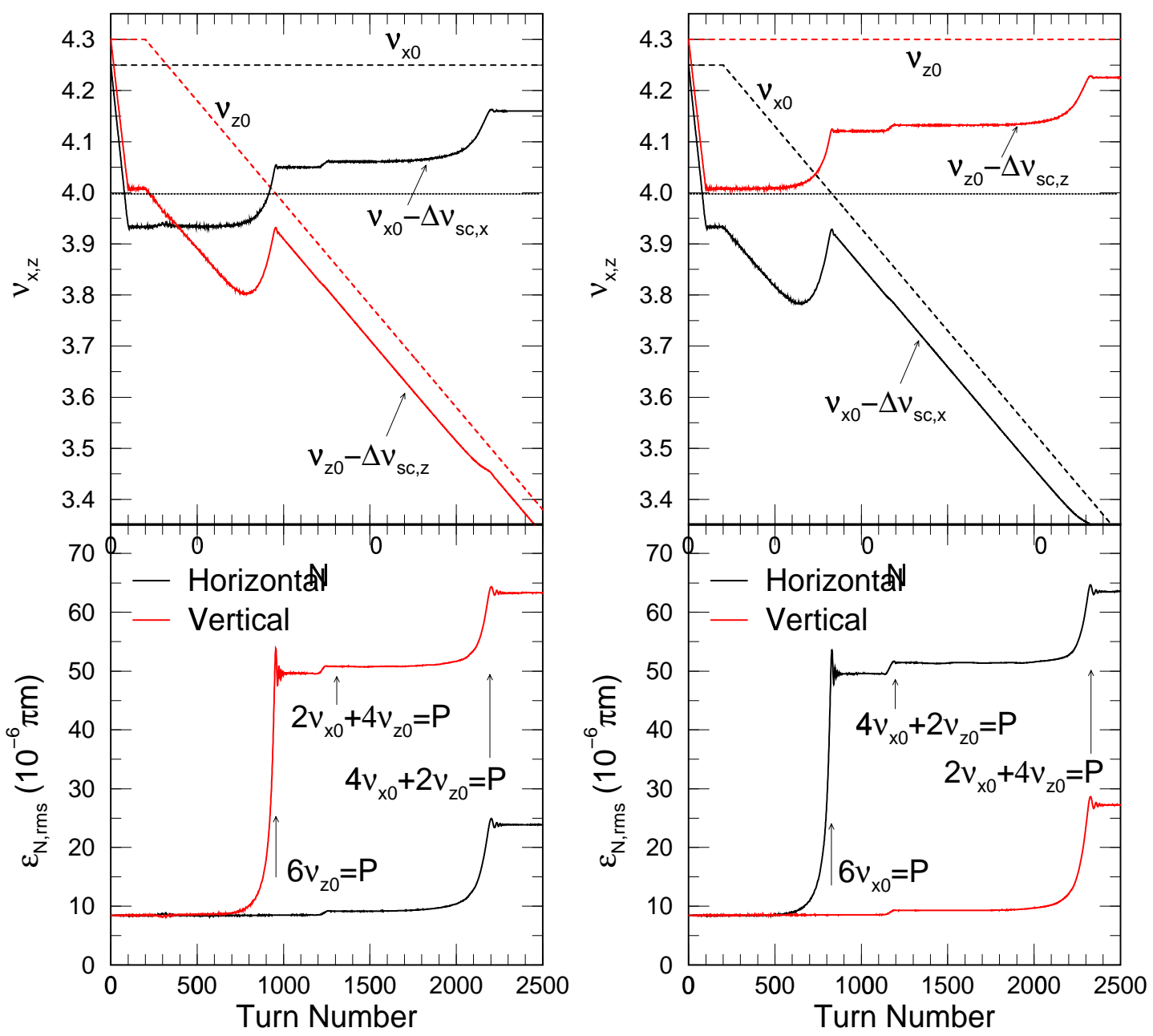

Figure 9. (Color) Left plots: Horizontal bare tune is kept constant at $\nu_{x 0}=4.25$, while the vertical bare tune is ramped downwards from $\nu_{z 0}=4.25$ at the rate of 0.0004 per turn starting from turn 200 after 100-turn injection at $2.0 \times 10^{11}$ per turn and bunching factor $B=0.25$. Systematic 6 th order resonances are crossed at turn 950 for $6 \nu_{z 0}=P$, turn 1262.5 for $2 \nu_{x 0}+4 \nu_{z 0}=P$, and turn 2200 for $4 \nu_{x 0}+2 \nu_{z 0}=P(P=24$ is the lattice periodicity). Note the large increase in emittance when the second sum resonance is crossed. Right plots are the same simulation, but with the vertical bare tune held constant at $\nu_{z 0}=4.30$ while the horizontal bare tune is ramped downwards from $\nu_{x 0}=4.25$ at the rate of 0.0004 per turn starting from turn 200. Systematic 6th order resonances are crossed at turn 825 for $6 \nu_{x 0}=P$, turn 1200 for $4 \nu_{x 0}+2 \nu_{z 0}=P$, and turn 1325 for $2 \nu_{x 0}+4 \nu_{z 0}=P$. Again large emittance increase is observed when the second sum resonance is crossed. 


\section{Fourth Order Parametric Resonance}

Octupoles are very often necessary to provide betatron tune spreads for the Landau damping of unwanted transverse instabilities. These octupoles are specially placed in the accelerator ring to maximize tune spreads and minimize nonlinear effects. As a result, the periodicity of the ring will be broken. Another source of octupole field comes from random nonlinear errors in the quadrupoles. They break the periodicity of the ring also. To mimic octupole effects, we simply add a single octupole in our model accelerator ring at the D-magnet of the last period.

\subsection{Resonance Strengths}

The potential of the octupole fields is

$$
V_{4}(x, z)=-\frac{1}{4 !} \frac{B^{\prime \prime \prime}}{B \rho}\left(x^{4}-6 x^{2} z^{2}\right),
$$

where $B \rho$ is the beam rigidity. After Floquet transformation, the terms responsible for the 4 th order resonances can be expressed in terms of action-angle variables in the form

$$
\begin{aligned}
V_{4}\left(J_{x}, J_{z}, \psi_{x}, \psi_{z}, \theta\right) \approx & -\frac{1}{R} \sum_{\ell}\left|G_{40 \ell}\right| J_{x}^{2} \cos \left(4 \psi_{x}-\ell \theta+\chi_{40 \ell}\right) \\
& -\frac{1}{R} \sum_{\ell}\left|G_{04 \ell}\right| J_{z}^{2} \cos \left(4 \psi_{z}-\ell \theta+\chi_{04 \ell}\right) \\
& -\frac{1}{R} \sum_{\ell, \pm}\left|G_{2 \pm 2 \ell}\right| J_{x} J_{z} \cos \left(2 \psi_{x} \pm 2 \psi_{z}-\ell \theta+\chi_{2 \pm 2 \ell}\right),
\end{aligned}
$$

where $\ell$ denotes any integer. In above $\left|G_{m n \ell}\right|$ and $\chi_{m n \ell}$ are the amplitude and phase of the resonance strength and they are computed by integrations around the accelerator ring over all the octupole fields:

$$
\begin{aligned}
G_{40 \ell} & =\frac{1}{96 \pi} \oint \frac{B^{\prime \prime \prime} \beta_{x}^{2}}{B \rho} \exp \left[j\left(4 \phi_{x}-4 \nu_{x} \theta+\ell \theta\right)\right] d s \\
G_{04 \ell} & =\frac{1}{96 \pi} \oint \frac{B^{\prime \prime \prime} \beta_{z}^{2}}{B \rho} \exp \left[j\left(4 \phi_{z}-4 \nu_{z} \theta+\ell \theta\right)\right] d s \\
G_{2 \pm 2 \ell} & =-\frac{1}{16 \pi} \oint \frac{B^{\prime \prime \prime} \beta_{x} \beta_{z}}{B \rho} \exp \left\{j\left[2 \phi_{x} \pm \phi_{z}-\left(2 \nu_{x} \pm 2 \nu_{z}\right) \theta+\ell \theta\right]\right\} d s .
\end{aligned}
$$

These resonance strengths can be made dimensionless by introducing the reduced resonance strengths

$$
g_{m n \ell}=G_{m n \ell} \epsilon_{\mathrm{rms}}
$$

where $\epsilon_{\mathrm{rms}}$ is the unnormalized rms emittance of the beam. Across a thin octupole of length $\ell$, the change in horizontal and vertical divergences are given by

$$
\begin{aligned}
& \Delta x^{\prime}=\frac{1}{6} S_{4}\left(x^{3}-3 x z^{2}\right), \\
& \Delta z^{\prime}=\frac{1}{6} S_{4}\left(z^{3}-3 x^{2} z\right),
\end{aligned}
$$


where the octupole strength is defined as $S_{4}=B^{\prime \prime \prime} \ell / B \rho$. For our test beam that has a kinetic energy of $1 \mathrm{GeV}, S_{4}=50 \mathrm{~m}^{-3}$ corresponds to a pole tip field of $0.035 \mathrm{~T}$ if the octupole physical aperture is of radius $5 \mathrm{~cm}$ and the octupole is of length $1 \mathrm{~m}$. With the octupole placed at a D-magnet with $\beta_{x}=6.3 \mathrm{~m}, \beta_{z}=21.4 \mathrm{~m}$, the resonance strengths at $\epsilon_{\mathrm{rms}}=4.61 \times 10^{-6} \pi \mathrm{m}$ (normalized $95 \%$ emittance $\epsilon_{\mathrm{N}}=50 \times 10^{-6} \pi \mathrm{m}$ ) are $\left|g_{40 \ell}\right|=3.03 \times 10^{-5},\left|g_{04 \ell}\right|=3.50 \times 10^{-4},\left|g_{2 \pm 2 \ell}\right|=1.03 \times 10^{-4}$. Since $\left|g_{40 \ell}\right|$ and $\left|g_{2 \pm 2 \ell}\right|$ are very much smaller, only the resonance $4 \nu_{z 0}=\ell$ will be studied here.

\subsection{Tracking}

Tracking is performed with 4000 macro-particles for 1200 turns. A larger number of macro-particles are used here because we noticed spurious emittance growths at a small number of macro-particles. Figure 10 shows a sample tracking with 70-turn injection of $4 \times 10^{11}$ protons each at bare tunes $\nu_{x 0}=6.95$ and $\nu_{z 0}=6.80$. Starting from turn 200 , both tunes were ramped downward linearly at the rate of 0.0005 per turn. The top-left plot shows both the bare tunes (dashes) and the space-charge depressed tunes (thick dots) as functions of turn number, while the bottom-left plot show the evolution of normalized rms emittances. The octupole strength is $S_{4}=20 \mathrm{~m}^{-3}$. We first see a split in horizontal and vertical emittances during injection, which signals Montague resonance, although the injection has been tailored to be at equal emittances. There is a vertical emittance growth near the $4 \nu_{z 0}=27$ resonance at turn 300 . This is verified by the vertical phase space plot (of turn 270) at the top-right with the four-arm-like structure. The next one encountered is the $2 \nu_{x 0}+2 \nu_{z 0}=27$ sum resonance at turn 450. Then the vertical hits the half-integer resonance $2 \nu_{z 0}=13$ at turn 800 , which is verified by the vertical phase space plot (of turn 780) at the bottom right, where we see particles streaming out in two directions. Then, there is another 4th order sum resonance $2 \nu_{x 0}+2 \nu_{z 0}=26$ at turn 950 followed by another half-integer resonance $2 \nu_{x 0}=13$ at turn 1100 . The 4 th order resonance in the horizontal plane $4 \nu_{x 0}=27$ should occur near turn 600, but it has almost been invisible, probably a result of the small horizontal betatron function at the octupole $\left(\beta_{x, D}=6.3 \mathrm{~m}\right)$. It is important to point out that the resonances crossed and the emittance growths always occur some revolution turns before the turn numbers suggested by the bare tunes. For example, $4 \nu_{z 0}=27$ predicts a resonance at turn 300, but the actual emittance growth peaks some 20 turns before that. The shifting of the resonance position comes from the depression of the tunes in the presence of the space-charge force. These resonances are coherent resonances of the beam envelope. Their references here by the bare tunes are just for the sake of convenience.

Unlike the systematic resonances studied in the previous sections, there has been severe beam loss of $6.2 \%$ during the 1200-turn tracking. The loss can be associated with the crossing of each of the resonances with the exception of, maybe, the first one. In the simulation, when the action exceeds a certain pre-determined value, the particle is defined as lost and is removed from the simulation. The computation of emittances 

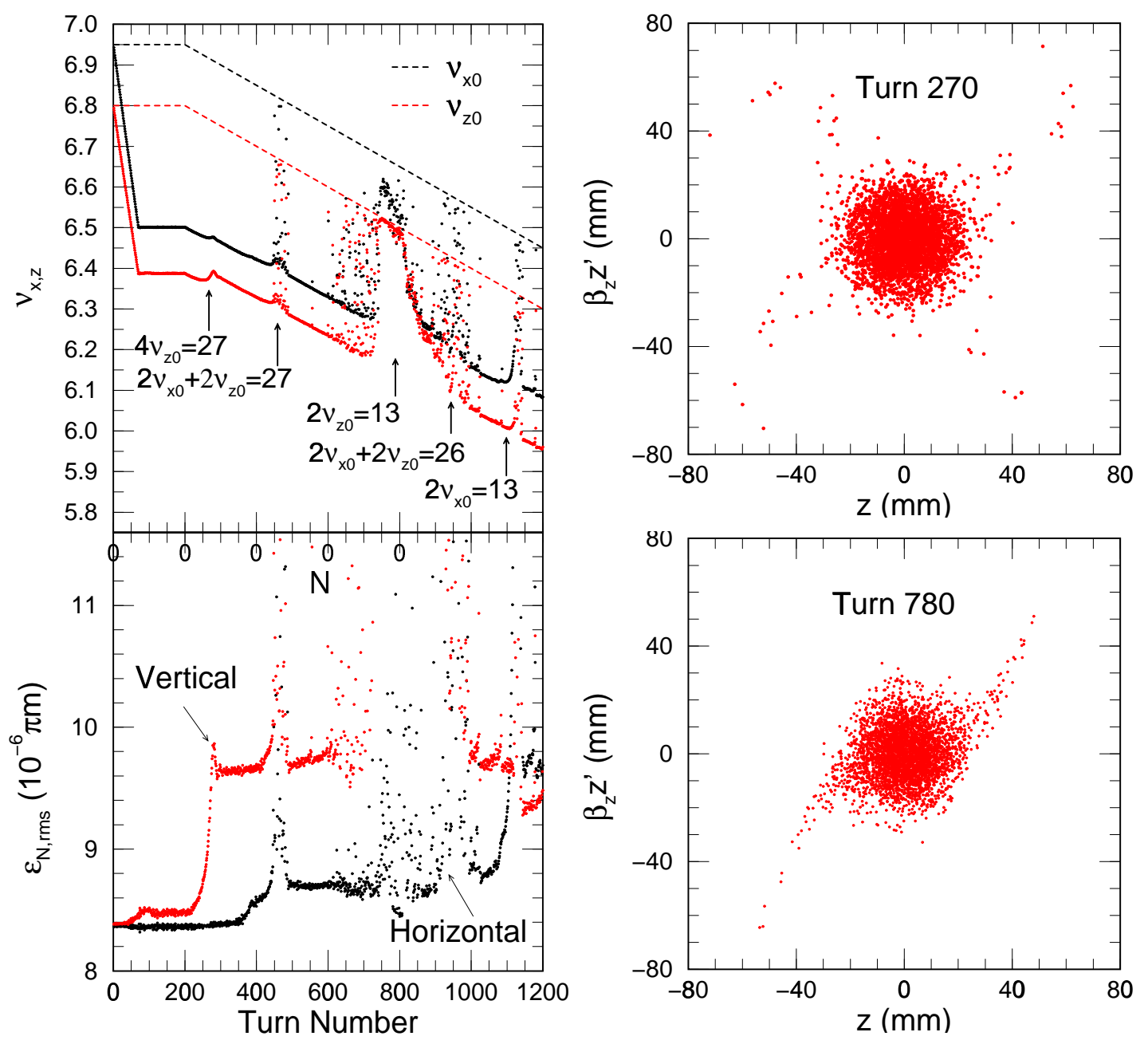

Figure 10. (Color) Top-left: After 70-turn injection at $4 \times 10^{11}$ per turn and bunching factor $B=0.25$, bare tunes are ramped downwards from $\left(\nu_{x 0}, \nu_{z 0}\right)=(6.95,6.80)$ starting at turn 200 at the rate of $-d \nu_{x 0, z 0} / d n=0.0005$. Octupole driven parametric resonances $4 \nu_{z 0}=27$ and $2 \nu_{x 0}+2 \nu_{z 0}=27$ are crossed at turns 300 and 450, respectively. Half-integer resonance is crossed in the vertical plane at turn 600 . The bare tunes are shown in dashes while the tunes depressed by linear space charge in thick dots. Bottom-left: Emittance growths are observed when the resonances are crossed. Heavy beam loss is observed. Right: vertical phase-space distributions at turn 270 (top) and 780 (bottom), demonstrating the crossing of $4 \nu_{z 0}=27$ and $2 \nu_{z 0}=13$, respectively. 
might become inaccurate in the presence of particle loss. This is because particles outside a core are sometimes pushed outward by the nonlinear force and become lost leaving behind a core that might have emittances that are rather small. This explains why emittances appear to decrease in the emittance plot in Fig. 10. In our study of emittance growth across the resonance $4 \nu_{z 0}=27$, we will include only those simulations where no particle loss is recorded across the resonance. This also explains why we are not able to have octupole strength stronger than $S_{4} \approx 50 \mathrm{~m}^{-3}$.

There are three parameters in the study of emittance growth crossing the octupole driven resonance $4 \nu_{z 0}=27$ : the beam intensity, the octupole strength, and the tuneramp rate. For the beam intensity, we study simulations of 70-turn injection with $1 \times 10^{11}, 2 \times 10^{11}, 3 \times 10^{11}$, and $4 \times 10^{11}$ protons per turns. They correspond to bunch intensity $8.33 \times 10^{10}, 16.7 \times 10^{10}, 25.0 \times 10^{10}$, and $33.3 \times 10^{10}$. The bunching factor and the beam kinetic energy have always been chosen to be $B=0.25$ and $1 \mathrm{GeV}$, respectively. At the normalized $95 \%$ emittances of $50 \times 10^{-6} \pi \mathrm{m}$, the corresponding linear spacecharge tune shifts are $\left(\Delta \nu_{s c, x}, \Delta \nu_{s c, z}\right)=(0.112,0.104),(0.224,0.207),(0.335,0.311)$, and $(0.447,0.414)$, respectively. The octupole strength is varied from $S_{4}=10 \mathrm{~m}^{-3}$ to $50 \mathrm{~m}^{-3}$. The tune-ramp rate is varied from $-d \nu / d n=0.00005$ to 0.006 .

\subsection{Scaling}

The simulation results for the four beam intensities are depicted in Fig. 11 and 12 as log-log plots. When the EGF is slightly larger than unity, the log-log plots show linear relations, implying that a scaling law relationship between the EGF and the tune-ramp rate, i.e., $\mathrm{EGF}=(-d \nu / d n)^{-a}$, where $a$ is a constant depending on the resonance strength and the space-charge tune shift. Here, $a$ varies from -0.35 to -0.65 , not by so much as for the 6th order systematic resonance. We extend this linear relationship to cut the $-d \nu_{z} / d n$-axis to obtain the critical tune-ramp rate. In general at these critical tune-ramp rates, EFG is $\lesssim 1.3$.

For each bunch intensity, the critical tune-ramp rate is plotted $\boldsymbol{\Phi}$ in Fig 13 as a function of the resonance strength $\left|g_{04 \ell}\right|$. The error bars reflect the uncertainty of the linear relationships of the plots in Figs. 11 and 12 and their intercepts on the $-d \nu_{z} / d n$ axis. It is evident that the data in Fig. 13 can be fitted linearly within the error bars. However, this may not be in contradiction to Fig. 7, because the resonance strengths $\left|g_{04 \ell}\right|$ here are very small compared with the $\left|g_{04 P}\right|$ in Fig. 7. Figure 13 provides some guidelines for the design of FFAGs. For a given linear space-charge tune shift of the beam and a given octupole strength, it tells us the minimum rate of crossing the 4th order resonance in order that the emittance will not grow by an intolerable amount.

I In a presentation of this paper to the 18th International Cyclotron Conference, the reduced resonance strength $g_{04} \ell$ has been incorrectly normalized to the normalized $95 \%$ emittance. As a result, the horizontal scale of Fig. 7 in Ref. [6] has been incorrect by the factor $6 \gamma \beta=10.85$. 

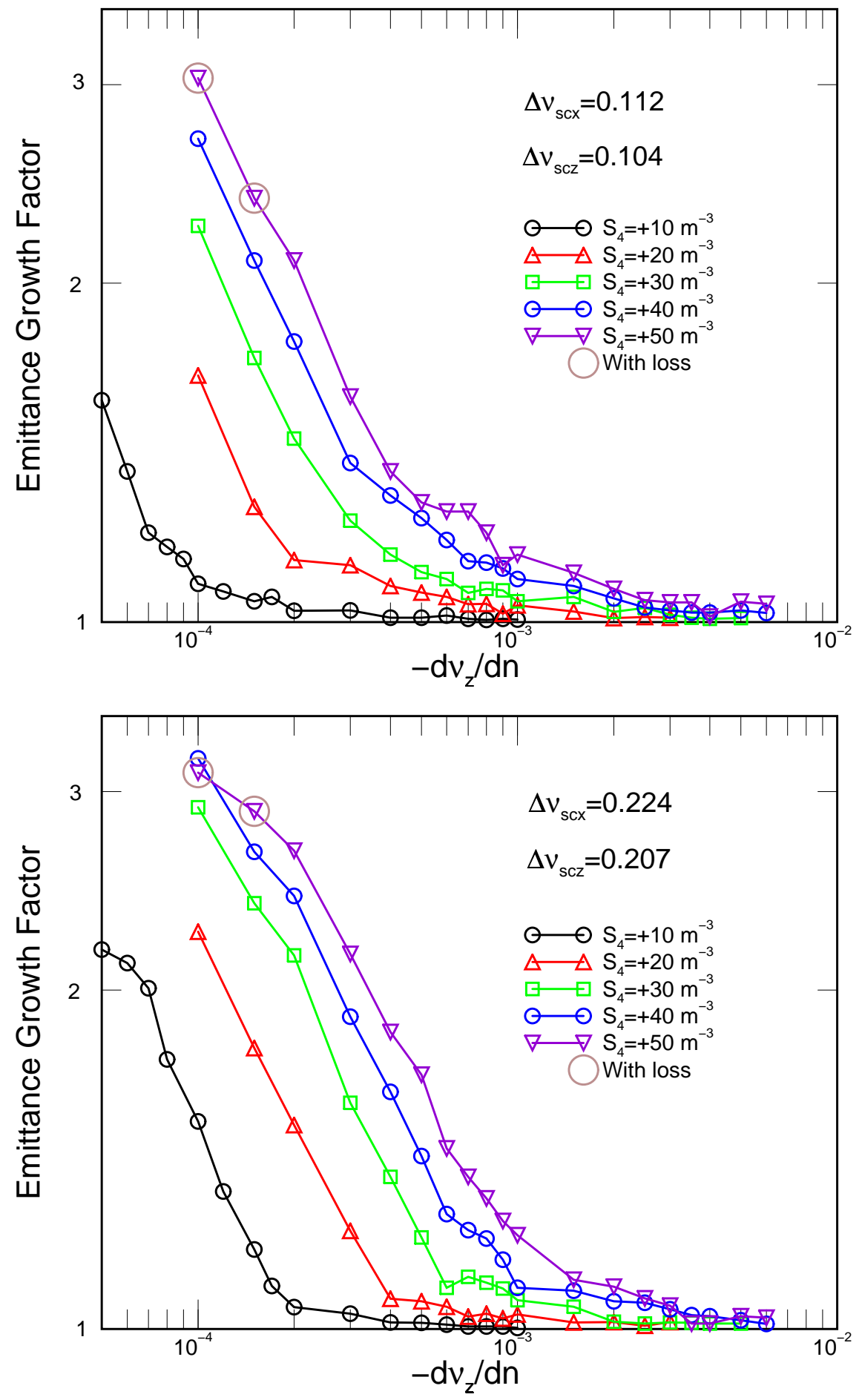

Figure 11. (Color) Emittance growth factors across the 4th order resonances $4 \nu_{x 0}=27$ driven by an octupole as functions of tune-ramp rate for various octupole strengths. The bunch intensity is $8.33 \times 10^{10}$ (top) and $16.7 \times 10^{10}$ (bottom) at the bunching factor of $B=0.25$ after 70 -turn injection. Data encircled involve particle loss and are discarded in the critical tune-ramp-rate analysis. 
Emittance growth scaling laws in resonance crossing
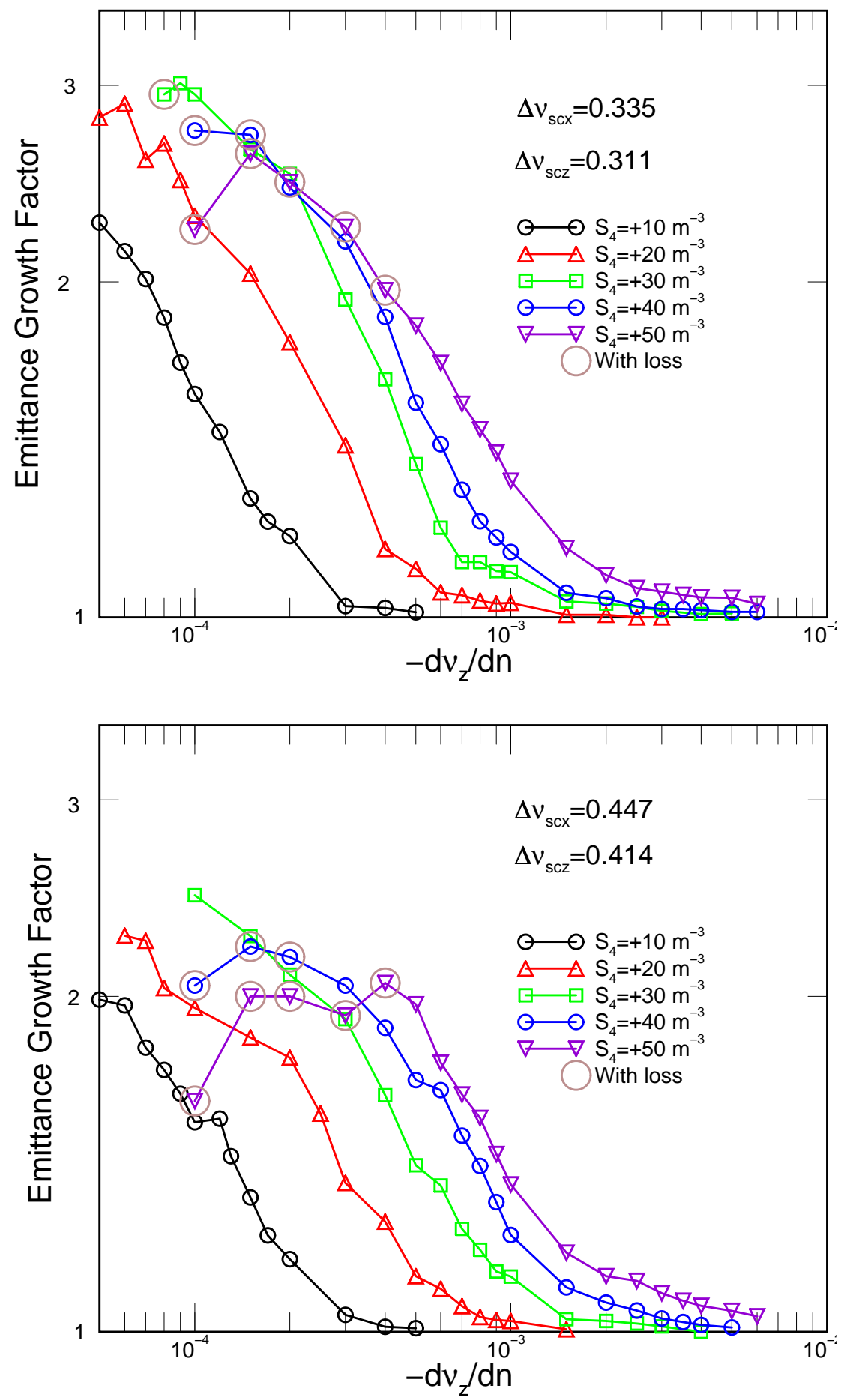

Figure 12. (Color) Emittance growth factors across the 4th order resonances $4 \nu_{x 0}=27$ driven by an octupole as functions of tune ramp rate for various octupole strengths. The bunch intensity is $25.0 \times 10^{10}$ (top) and $33.3 \times 10^{10}$ (bottom) at the bunching factor of $B=0.25$ after 70-turn injection. Data encircled involve particle loss and are discarded in the critical tune-ramp-rate analysis. 


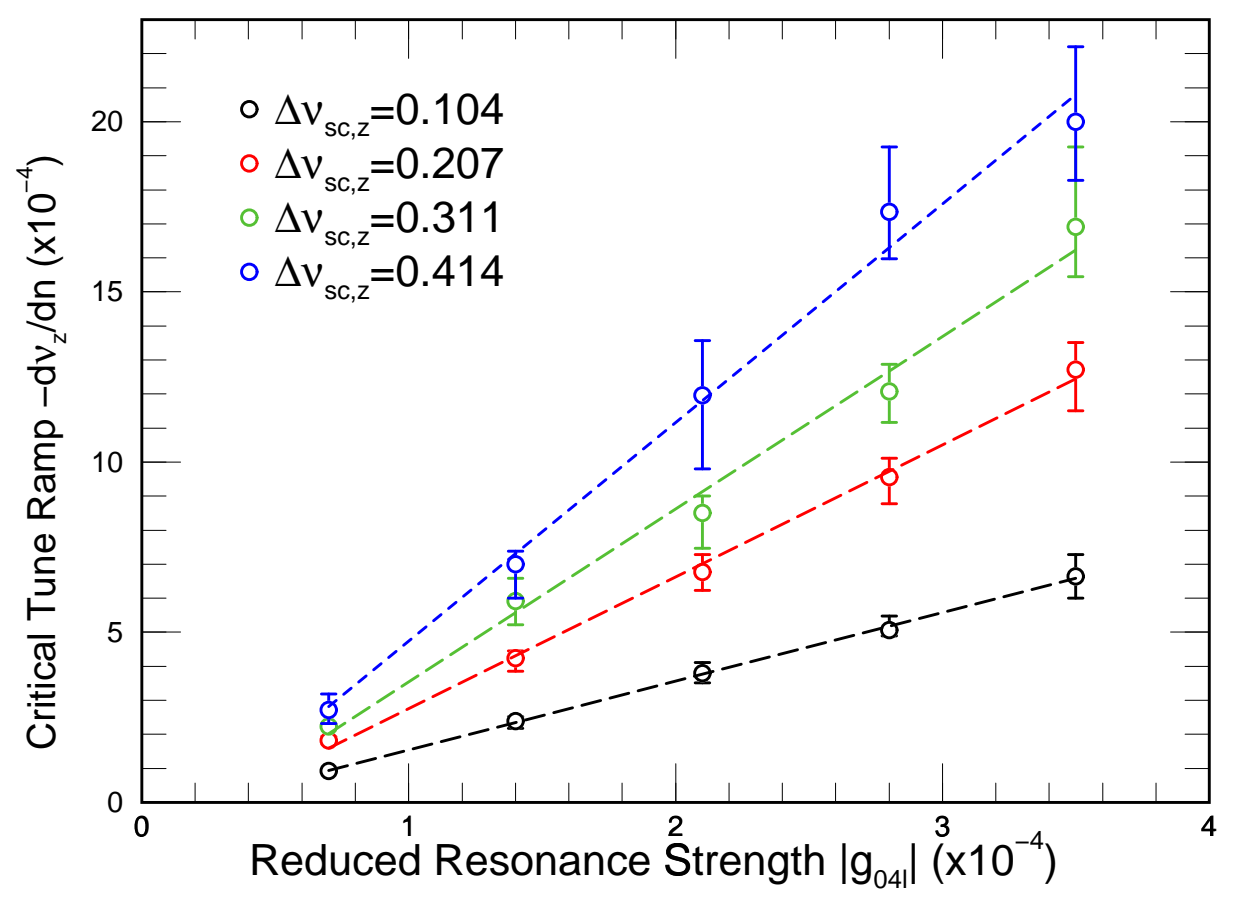

Figure 13. (Color) Critical tune-ramp rates across the octupole driven 4th order resonances $4 \nu_{z 0}=27$ are plotted as functions of reduced resonance strength $g_{04 \ell}$ for various bunch intensities. Dashed lines are linear fits to the data.

\section{Conclusions}

For a ramping cycle in a non-scaling FFAG, betatron-tune crossing of many units is unavoidable. The beam motion may cross many resonances in the transverse phase spaces. We have performed simulations to study emittance growths on crossing systematic 6th order resonances driven by the space-charge force as well as the parametric 4th order resonances driven by an octupole-like magnetic field.

Just as in the investigation in Refs. [4] and [5], for the 6th order resonances $6 \nu_{x 0}=P$ and $6 \nu_{z 0}=P$, we discover linear scaling relationships in the EGFs and tune-ramp rates when the two are displayed in a log-log plot. Critical tune-ramp rates are then determined as functions of space-charge tune shifts and reduced resonance strengths which are lattice dependent. These functions can serve as a guide for the design of nonscaling FFAGs so that emittance growths across these resonances can be kept within toleration.

For a lattice similar to that of the Fermilab Booster, the critical tune-ramp rates for linear space-charge tune shifts of $\sim 0.31$ have been found to be of the order 0.011 per turn. This means that, when the particle kinetic energy is $1 \mathrm{GeV}$ and the betatron tune is $\nu_{x 0}=4$, the energy gain per revolution should be of the order of $10 \mathrm{MeV}$, which is very large for the low frequency rf systems. For this reason, phase advance per cell should not hit $\frac{\pi}{2}$ for the 4 th order systematic resonance and not cross $\frac{\pi}{3}$ for the 6 th order 
systematic resonance during the FFAG ramp cycle.

For the parametric 4 th order resonance $4 \nu_{z 0}=\ell$, we also find linear scaling relations in the log-log plot of the EGF versus tune-ramp rate. So far we have been able to work with a small reduced resonance strength $\left(\left|g_{04 \ell}\right| \lesssim 3.5 \times 10^{-4}\right)$, because severe particle loss will occur when the resonance strength becomes larger. The corresponding critical tune-ramp rate is $\sim 2 \times 10^{-3}$ per turn for linear space-charge tune shift $\Delta \nu_{s c, z}=0.41$.

If the protons in the FFAG are replaced by muons, such design restrictions do not apply. This is because the space-charge tune shift or space-charge force at the same particle energy will be reduced by $m_{p}^{2} / m_{\mu}^{2}=78.9$ times and the EGFs across resonances will be greatly reduced accordingly.

Our results show that the critical tune-ramp rate versus reduced resonance strength can be fitted by straight lines, both for the systematic 6th order resonances and the parametric 4th order resonance. On the other hand, the result of Refs. [4] and [5] gives obviously nonlinear relationship for the systematic 4th order resonance. However, the 6 th order resonances are intrinsically much weaker than the 4 th. In fact, the maximum $g_{60 P}$ and $g_{06 P}$ in the space charge potential have strengths less than one-sixth of the maximum $g_{40 P}$ and $g_{04 P}$ presented in Refs. [4] and [5]. The maximum critical tuneramp rate is also about one-fifth smaller. The reduced resonance strengths $g_{04 \ell}$ for the parametric 4th order resonance driven by octupole are even much smaller. It is easy to see that for the smaller ranges of critical tune-ramp rate and $g_{40 P}$ and $g_{04 P}$ in Refs. [4] and [5], the data can also be fitted with linear straight lines. It will be interesting to perform further study with larger resonance strength for the systematic 6th order resonance to see whether the linear relationships between critical tune-ramp rate and reduced resonance strength continue to hold or break down.

An effective space-charge force has been employed in all the simulations. This force has the merit that it is simple and analytic so that the simulations can be sped up. However, this space charge force has the disadvantage that it is not derivable from a potential, implying that Liouville's theorem is only approximately valid provided that the nonlinear space-charge sum and difference resonances are not crossed. This explains why emittance growths across those resonances have not been investigated in this paper.

Although the space-charge force is two-dimensional and is dependent on the beam radii in both transverse directions, we find that the emittance is conserved when no resonance is crossed. Thus our model is a reasonable approximation for the study of 1D resonance crossing due to the space-charge force. The next most important task will be to solve the singularity issue of the exact space-charge force, which is expressed in terms of the complex error function, so that it can be applied to the simulations. Otherwise, we need to devise another effective space-charge force that is derivable from a potential.

\section{References}

[1] K.R. Symon, D.W. Kerst, L.W. Jones, L.J. Laslett, and K.M. Terwilliger, Fixed-Field AlternatingGradient Particle Accelerators, Phys. Rev. 103, 1837 (1956). 
$[2]$ D. Trbojevic, E.D. Courant, and A.A. Garren, FFAG lattice without Opposite bends, Muon Colliders at $10 \mathrm{TeV}$ and $100 \mathrm{TeV}$ HEMC '99 Workshop, Colliders and Collider Physics at the Highest Energies, Montauk, New York, September 27-October 1, 1999, AIP Conference Proceedings, pp. 333-338; C. Johnstone, W. Wan, and A. Garren, Fixed Field Circular Accelerator Designs, Proc. 1999 Particle Accelerator Conference, New York, 1999, p. 3068.

[3] A.G. Ruggiero, FFAG Accelerator Proton Driver for Neutrino Factory, Brookhaven Report C$\mathrm{A} / \mathrm{AP} / \# 219,2005$.

[4] S.Y. Lee, Fundamental Limit of Nonscaling Fixed-Field Alternating-Gradient Accelerators, Phys. Rev. Lett. 97, 104801 (2008).

[5] S.Y. Lee, G. Franchetti, I. Hofmann, F. Wang, and L. Yang, Emittance Growth Mechanism for Space-Charge Dominated Beams in Fixed Field Alternating Gradient and Proton Driver Rings, New J. Phys. 8, 291 (2006).

[6] K.Y. Ng, X. Pang, F. Wang, X. Wang, and S.Y. Lee, Enittance Growths in Resonance Crossing at FFAGs, Proc. 18th Int. Conf. on Cyclotrons and their Applications, Giardini Naxos, Messina, Italy, Sep. 30-Oct. 5, 2007. 